\title{
Forschungsfeld experimentelle Wirtschaftswissenschaften
}

Experimente bilden ein klassisches Verfahren der empirischen Sozialforschung. In den unterschiedlichen sozialwissenschaftlichen Disziplinen ist der Einsatz von Experimenten dabei verschiedentlich stark ausgeprägt. Während Experimente beispielsweise in der Geschichtswissenschaft kaum genutzt werden und in der Soziologie und der Politikwissenschaft im Vergleich zu anderen Verfahren weniger häufig Verwendung finden, zeigt sich beispielsweise in den Wirtschaftswissenschaften und insbesondere im Bereich der Verhaltensökonomie eine deutliche Affinität für dieses Verfahren der empirischen Forschung. Hier insbesondere in der Form des Laborexperiments. Für die intensive Auseinandersetzung mit dem Phänomen des wirtschaftswissenschaftlichen Laborexperiments und seiner Eigengesetzlichkeit ist es hilfreich, sich in einem ersten Schritt mit den allgemeinen methodologischen Grundlagen des Experiments in den Sozialwissenschaften auseinanderzusetzen. Dieser Abschnitt widmet sich deshalb der Einführung zentraler Konzepte und methodologischer Ansprüche des experimentellen Forschungsdesigns, wie beispielsweise der Isolation, Kontrolle und Manipulation hypothesenrelevanter Variablen, dem Parallelgruppenmodell, den Verfahren der Randomisierung und Anonymisierung sowie dem Anspruch einer Kausalitätsanalyse, welche mit dem experimentellen Vorgehen verbundenen wird. Zur besseren Einordnung des Laborexperiments werden im Anschluss die drei (in der quantitativen Forschung) typischen Formen des Experiments - Laborexperiment, Feldexperiment und natürliches Experiment - kurz voneinander abgrenzt. Nach dieser Thematisierung der Grundlagen des experimentellen Forschungsdesigns erfolgt im Anschluss eine kurze Einführung in das Feld der experimentellen Wirtschaftsforschung und die feldinternen Begründungen für die Anwendung von experimentellen Datenerhebungsverfahren.

J. Haus, Das ökonomische Laboratop, Wissen, Kommunikation und Gesellschaft, https://doi.org/10.1007/978-3-658-33019-4_2 


\subsection{Das Experiment in den Sozialwissenschaften}

Im Unterschied zu naturwissenschaftlichen Experimenten sind die Forschungsgegenstände sozialwissenschaftlicher Experimente Menschen oder spezifische menschliche Handlungen, Verhaltensweisen und Entscheidungen. Wie die nachfolgenden Ausführungen zeigen werden, gehen mit der standardisierten Untersuchung menschlichen Verhaltens, Handelns und Entscheidens eine Reihe von Herausforderungen durch die Vielfalt potenzieller Reaktivitätsprobleme einher, denen im Rahmen von sozialwissenschaftlichen Experimenten auf unterschiedliche Weise begegnet wird.

Wie Ekkart Zimmermann (1972: 34) in seiner grundlegenden Beschreibung festhält, handelt es sich bei einem sozialwissenschaftlichen Experiment ,nicht um eine besondere Form der Datenerhebung, sondern um eine spezielle Art der Untersuchungsplanung [...] Wichtig ist beim Experiment der manipulative Eingriff in die soziale Realität. Andere Techniken der Sozialforschung sind dagegen durch bloße Auswahl bestimmter Aspekte der Realität gekennzeichnet". In einem ersten Schritt gilt es anzuerkennen, dass es sich bei einem Experiment nicht einfach um eine Methode der Datenerhebung handelt, sondern um ein Forschungsdesign. Das Experiment bezeichnet somit eine besondere Form der Anordnung und Durchführung einer empirischen Untersuchung. Der manipulative Eingriff und damit die aktive Veränderung und Variation der Erhebungssituation ist dabei das zentrale Moment des experimentellen Forschungsdesigns. Dieses Vorgehen wird auch als Prinzip der aktiven Erfahrung bezeichnet und wurde bereits im 17. Jahrhundert unter dem Einfluss von Francis Bacon in die Naturwissenschaft eingeführt (Eifler 2014: 195).

Nicht nur in den Natur-, sondern auch in den Sozialwissenschaften gilt das Experiment als das klassische Design zur Untersuchung von Kausalzusammenhängen, welche von einem Ursache-Wirkungs-Prinzip ausgehen. Die in einem Experiment betrachteten Fragestellung folgen dabei einem kausalanalytischen Muster: In welcher Weise und Stärke wirkt sich A (Ursache) auf B (Folge) aus? Grundlage für dieses kausalanalytische Argumentationsschema bildet ein analytisch-nomologisches Wissenschaftsverständnis, welches davon ausgeht, dass es in der sozialen Realität grundlegende Gesetzmäßigkeiten gibt. Diese und ihre zugehörigen Eigenschaften der sozialen Realität besitzen allgemeine Geltung. Mittels Hypothesen werden nun Vermutungen über diese Zusammenhänge aufgestellt. Im Folgenden werden Ausschnitte der sozialen Welt identifiziert, in denen die Hypothesen getestet werden können, um im Anschluss aus den Ergebnissen abzuleiten, ob sich die Hypothesen verifizieren oder falsifizieren lassen beziehungsweise ob sie umformuliert werden müssen (Kromrey 2009: 20 f.). 
Im methodischen Diskurs werden an dieser Stelle in der Regel die Begriffe der abhängigen und unabhängigen Variablen genutzt. Die abhängige Variable bildet dabei das Phänomen, das in seiner Ausprägung durch die Veränderung der unabhängigen Variablen, das heißt des isolierten Ursachenfaktors, bestimmt wird.

\section{Isolation und Kontrolle}

Wie oben dargestellt, bildet die gezielte Manipulation des unabhängigen Einflussfaktors den Kern des klassischen experimentellen Vorgehens. In einem klassischen Experiment wird dabei stets angestrebt, durch Isolation und Kontrolle der hypothesenrelevanten Variablen Untersuchungsbedingungen $\mathrm{zu}$ schaffen, unter welchen nur das Ursache-Wirkungs-Prinzip als Erklärung für die Veränderung des Phänomens herangezogen werden kann (Behnke et al. 2010: 42). Zugleich gilt es dafür Sorge zu tragen, dass die Art und Intensität der durch die Hypothese unterstellten Wirkung eindeutig festgestellt und (quantitativ) gemessen werden kann. Die Grundlagen dieser idealtypischen Konzeption des experimentellen Forschungsdesigns sollen nun kurz dargestellt werden (Behnke et al. 2010; Eifler 2014; Zimmermann 1984, 2015).

Der Aspekt der Isolation bezieht sich in diesem Zusammenhang auf den notwendigen Anspruch, den Ursachenfaktor, das heißt die als ursächlich relevant angenommene Einflussgröße (unabhängige Variable), im Rahmen des beobachteten Phänomens von allen anderen möglichen Einflussfaktoren abzuschirmen. Der Aspekt der Kontrolle bezieht sich im Unterschied dazu auf die Annahme, dass der Einfluss des Ursachenfaktors vom Forschenden gezielt in die Situation eingeführt und auf systematische Weise variiert werden kann. Damit verbunden ist der Anspruch, dass der Forschende bestimmt, in welchem Maße die Wirkung des angenommenen Ursachenfaktors zugelassen wird. Darüber hinaus soll der manipulierte Ursachenfaktor nicht erst stark zeitverzögert wirken, sondern muss dies im Rahmen der kontrollierten Erhebungssituation tun, um in einer Messung registriert werden zu können. Da angenommen wird, dass alle anderen Einflussfaktoren ausgeschaltet sind, wird aufbauend auf der gezielten Variation des Ursachenfaktors auf den Kausalzusammenhang zwischen diesem und dem beobachteten Phänomen geschlossen. Dies wird dadurch begründet, dass unter Annahme des Konstanthaltens der restlichen Versuchsbedingungen (CeterisParibus-Klausel (siehe unten)) nur die Veränderung des isolierten Einflussfaktors den Grund für die Veränderung des Phänomens darstellen kann.

\section{Parallelgruppenmodell}

In seiner strengen Form orientiert sich das experimentelle Vorgehen dabei an den klassischen Naturwissenschaften wie der Physik und Biologie und den dort 
durchgeführten Experimenten. Das Experiment gilt in diesen Disziplinen als das zentrale wissenschaftliche Vorgehen für den Test von Hypothesen und Theorien. Die grundlegende Form des experimentellen Vorgehens besteht in den Naturwissenschaften darin, dass Forschende absichtlich einen Gegenstand durch einen Eingriff manipulieren, um so eine Wirkung zu erzeugen. Tritt eine Veränderung des Gegenstandes ein, so wird die Wirkung des aktiven Eingriffs des Forschenden als Ursache für diese Veränderung betrachtet. In naturwissenschaftlichen Experimenten erfolgt üblicherweise eine Vorhermessung der interessierenden Variablen, bevor sie dem manipulierenden Einfluss des Stimulus ausgesetzt werden. Auf diese Weise wird der Ausgangs- oder Normalzustand des Gegenstandes hinsichtlich der Ausprägung der interessierenden Aspekte bestimmt. Im Anschluss an die Manipulation des Ursachenfaktors erfolgt eine erneute Messung dieser Aspekte, die mit der ersten Messung verglichen wird. Die Interpretation der Wirkung eines manipulierenden Eingriffs hängt dabei von der Bestimmung der Normalsituation ab, mit der das Resultat des experimentellen Eingriffs verglichen wird. In den Sozialwissenschaften liegen solche Normalsituationen für das Auftreten sozialer Forschungsgenstände in der Regel nicht vor. Zudem können die Veränderungen eines Forschungsgegenstands häufig nicht eindeutig auf die Wirkung des experimentellen Eingriffs zurückgeführt werden, da auch andere Einflussfaktoren als eine potenzielle Erklärung der Veränderung nicht ausgeschlossen werden können (Eifler 2014: 197). Die Validität der Untersuchung und des unterstellten Kausalzusammenhangs hängt dabei stark von der Bewältigung dieser Herausforderungen des Messvergleichs ab. Im Design sozialwissenschaftlicher Experimente wird dieser Herausforderung durch eine spezifische Form der Gruppenbildung begegnet (Berger \& Wolbring 2015: 41), welche auch als Parallelgruppenmodell bezeichnet wird (Eifler 2014: 200). In sozialwissenschaftlichen Experimenten werden Menschen - als Versuchspersonen oder ProbandInnen mit den unterschiedlichen Versuchsanordnungen (Treatments) konfrontiert. Ein grundlegendes Designelement des experimentellen Forschungsdesigns besteht darin, die an der Forschung beteiligten Personen gezielt in zwei Gruppen einzuteilen: die Kontrollgruppe und die Experimental- bzw. Versuchsgruppe. Es wird von der Annahme ausgegangen, dass vor der Datenerhebung beide Gruppen in der Verteilung der interessierenden abhängigen Variablen übereinstimmen. Bei der Versuchsgruppe wird untersucht, welche Auswirkung das Treatment, das heißt der Einsatz des manipulativen Eingriffs auf die Ausprägung der abhängigen Variablen zeigt. Bei der Kontrollgruppe hingegen wird kein manipulativer Eingriff vorgenommen und die abhängige Variable in ihrer Ausprägung gemessen. Sollte sich ein Unterschied zwischen den beiden Gruppen zeigen, so wird dieser auf die Veränderung des Einflussfaktors (der unabhängigen Variablen) zurückgeführt. 
Diese Differenz wird auch als Treatment-Effekt bezeichnet. Das experimentelle Untersuchungsdesign ist dabei jedoch nicht auf den Vergleich zwischen zwei Untersuchungsgruppen beschränkt. Häufig werden in einer Serie von Erhebungen mehrere Versuchsanordnungen (Treatment-Variationen) getestet, in denen der angenommene Einflussfaktor in unterschiedlicher Weise manipuliert wird. Es werden somit mehrere Versuchsgruppen miteinander und mit der Kontrollgruppe verglichen.

\section{Konstanthalten der Randbedingungen}

Die Isolation und Kontrolle der hypothesenrelevanten Variablen sind zentrale Herausforderungen in der Untersuchungsplanung sozialwissenschaftlicher Experimente. Sie sind zugleich die maßgebliche Grundlage dafür, dass der interessierende Kausalzusammenhang überprüft werden kann. Die Isolation und Kontrolle der zu testenden Variablen meint dabei jedoch nicht, dass sie nicht von anderen Randbedingungen umgeben sein würden. Gemeint ist vielmehr, dass alle weiteren Randbedingungen konstant gehalten werden und nur die interessierende Einflussgröße verändert wird. Die Notwendigkeit des Konstanthaltens der Randbedingungen schließt dabei an die „Methode der Differenz“ von John Stuart Mill (1982) an. Sie bezieht sich einerseits auf Beobachtungen, in denen das interessierende Phänomen einmal festgestellt werden konnte, und andererseits auf solche, in denen es nicht auftrat. Sind diese beiden Fälle bis auf eine Variable gleich, so wird darauf geschlossen, dass diese Variable die Ursache der untersuchten abhängigen Variablen ist. Diese Annahme entspricht dem Ursache-Wirkungs-Prinzip. Zugleich wird dabei jedoch auch abgeleitet, dass dieses Verfahren geeignet ist, um zu bestimmen, dass gewisse Faktoren keinen ursächlichen Einfluss auf das interessierende Phänomen haben. Liegen bestimmte Faktoren sowohl bei Auftreten des Phänomens als auch bei seiner Abwesenheit vor, so wird geschlussfolgert, dass diese in keinem ursächlichen Zusammenhang mit dem untersuchten Phänomen stehen (Zimmermann 1972: 25 f.). Das Konstanthalten der restlichen Untersuchungsbedingungen wird auch als Ceteris-Paribus-Klausel bezeichnet.

Damit die Schlussfolgerung eines Kausalzusammenhangs zwischen den untersuchten Variablen eindeutig ist, muss zudem die „SUTVA-Annahme“ (Stable Unit Treatment Value Assumption) (Rubin 1986) erfüllt sein. Diese besagt, dass die Versuchsbedingungen (und damit der wirkende Stimulus) auf jede der Versuchspersonen die gleiche kausale Wirkung ausüben muss, unabhängig von ihrer Zuteilung zur Kontroll- und Versuchsgruppe. Im Anschluss an Markus Gangl (2010) verweisen auch Roger Berger und Tobias Wolbring (2015: 43) drauf, dass die SUTVA-Annahme in sozialwissenschaftlichen Kontexten oft ein 
unrealistisches Ideal bleibt, da es im sozialen Prozess des Experiments zu vielfältigen Reaktivitätsproblemen kommen kann. Beispiele dafür bilden die sozialen Interaktionen zwischen den Versuchsgruppen oder eine ungleiche Informationsverteilung, die die Wirkung des Stimulus beeinflussen können. Im Unterschied zum physikalischen Reinraum und Untersuchungen im Vakuum ist es nicht möglich, die soziale Welt soweit zu reduzieren, dass sich einzelne Wirkfaktoren isolieren und abschirmen lassen.

Hinsichtlich der Isolier- und Manipulierbarkeit zwischen natur- und sozialwissenschaftlichen Untersuchungsgegenstände besteht also eine folgenreiche Differenz. Während die Herauslösung naturwissenschaftlicher Forschungsobjekte aus der realweltlichen Komplexität ihres Auftretens und die Kontrolle der beeinflussenden Aspekte durch eine geeignete Versuchsanordnung möglich erscheinen, ist sozialwissenschaftliche Forschung auf menschliche Subjekte bezogen. Diese sind soziale Wesen, die durch spezifische idiosynkratrische Erfahrungen geprägt wurden und sich ihre umgebenden Situationen deutend aneignen. Im Zuge der experimentellen Erhebung werden diese Individuen gleichartigen Bedingungen ausgesetzt. Sie werden dabei zu Datenlieferanten für die quantitativen Messungen in verschiedenen Versuchsanordnungen. Jede Versuchsperson liefert dabei eine Reihe von Daten, die im Anschluss an das Experiment für die statistische Analyse genutzt werden. Die Einflussfaktoren, die die angestrebte Messung des zu testenden Zusammenhangs ungewollt beeinflussen, werden als Störvariablen, Störfaktoren, Rauschen oder Noise bezeichnet. Es muss davon ausgegangen werden, dass stets Störvariablen vorliegen, die die angestrebte Isolation und Kontrolle der interessierenden Variablen gefährden.

\section{Kontrolltechniken}

Im Bereich der experimentellen Forschung in den Sozialwissenschaften haben sich eine Reihe von Standardverfahren etabliert, mit denen versucht wird, diesen potenziellen Verzerrungen durch die Störfaktoren zu begegnen. Die Anwendung dieser Verfahren insbesondere des Parallelgruppendesigns und der Randomisierung, ist mittlerweile so tradiert, dass sie selbst zu einem Kennzeichen für das experimentelle Forschungsdesign geworden sind. Die Soziologin Stefanie Eichler weist im Anschluss an den Psychologen Oswald Huber (2013: 104 f.) darauf hin, dass dabei grundlegend zwei Arten von Kontrolltechniken unterschieden werden können. Zum einen solche, die in der experimentellen Erhebungssituation selbst Anwendung finden und zum anderen solche, die auf die untersuchte Population oder Stichprobe gerichtet sind. Kontrolltechniken die in der Situation der Datenerhebung eingesetzt werden, zielen auf das Konstanthalten der Randbedingungen und das Eliminieren möglicher Störfaktoren. Durch ein Konstanthalten der 
Erhebungssituation wird angestrebt, die Varianz der Einflussfaktoren zu begrenzen und so auch die Wirkung, die diese auf das Ergebnis ausüben können. Dies ist für das Ergebnis der Experimente von entscheidendem Belang, da im Rahmen der Erhebung die Daten mehrerer Durchführungen einer Versuchsanordnung in der Analyse zusammengefasst werden. Dies ist jedoch nur zulässig, wenn von einem Konstanthalten der Randbedingungen ausgegangen werden kann und somit davon, dass es sich um die gleiche Versuchsanordnung handelt. Der Weg eine Gleichartigkeit der Erhebungssituation zu realisieren, wird in der umfangreichen Standardisierung vielfältiger Aspekte der Versuchsanordnung und des Ablaufs der Experimente gesehen. Zugleich werden gezielt Verfahren eingesetzt, um die experimentelle Erhebungssituation von bestimmten Störfaktoren abzuschirmen. In diesem Zusammenhang wird der Erhebung im Experimentallabor der Vorteil zugesprochen, dass dieser Raum eine besonders gute Abschirmung gegenüber externen Störeinflüssen, wie beispielsweise Lärm, Ablenkungen oder unvorhergesehene Zwischenfälle ermöglicht.

Auch die vielfach eingesetzten Anonymisierungstechniken können dabei als Kontrolltechnik gegenüber unerwünschten Störfaktoren betrachtet werden. Es handelt sich hierbei um Verfahren, in denen die Identität der Versuchspersonen und ihre Handlungen für andere Personen im Rahmen der Experimente verborgen bleiben (Franzen \& Pointner 2015). Die Verfahren zielen darauf ab, Verzerrungen der Ergebnisse zu verringern, die sich durch soziale Erwünschtheitseffekte ergeben könnten. Diese Techniken kommen besonders dann zum Einsatz, wenn mehrere Versuchspersonen im Rahmen einer Erhebung mitanderen interagieren und SpielerInnengruppen bilden. Es gibt dabei unterschiedliche Varianten der Anonymisierungsverfahren. Eine grundlegende Form der Anonymisierung besteht beispielsweise, wenn den Versuchspersonen in einer SpielerInnengruppe zwar mitgeteilt, dass sich die Gruppenmitglieder im Laborraum befinden und auch wie sie in einer Situation gehandelt oder gewählt haben, jedoch nicht um welche Personen im Raum es sich handelt. Im Unterschied dazu wird den ProbandInnen bei einfachblinden Versuchen (,single blind“) die Entscheidung ihrer SpielpartnerInnen nicht mitgeteilt. Sie werden in diesen Fällen nur über die Konsequenz der Entscheidung in Kenntnis gesetzt. Die ExperimentatorInnen haben hingegen Kenntnis von den Entscheidungen aller Beteiligten SpielpartnerInnen. Bei doppelblind Versuchen (,double blind“) können auch die ExperimentatorInnen den einzelnen Versuchspersonen keine Handlungen oder Ergebnisse zuordnen. In diesem Fall besteht somit sowohl zwischen den ProbandInnen als auch zwischen den Versuchspersonen und VersuchsleiterInnen Anonymität. 


\section{Randomisierung}

Um dem Ideal der Ausschaltung von verzerrenden Störeinflüssen auf das Ergebnis des experimentellen Eingriffs bestmöglich zu entsprechen, wird das Verfahren der Randomisierung angewendet. Dies bezeichnet die zufällige Zuordnung der Versuchspersonen zur Kontroll- und Versuchsgruppe mit dem Zweck, systematische Verzerrungen der Ergebnisse durch unbeobachtete und beobachtete Störvariablen auszuschalten. Die Orientierung an diesem Konzept geht auf den Statistiker Ronald Aylmer Fisher zurück (Hall 2007). Dieser zeigte durch eine Reihe von Experimenten in der Landwirtschaft den Vorteil der zufälligen Zuweisung auf. Den Ausgangspunkt bildete das grundlegende Problem, dass Ackerflächen unterschiedliche Bodenqualitäten aufweisen. Aus diesem Grund war es nicht möglich, die Qualität unterschiedlicher Saatgutvarianten eindeutig zu bestimmen. In der zufälligen Verteilung unterschiedlicher Saatguttypen auf verschiedene Ackerparzellen erkannte Fisher einen Weg, die Beschaffenheit des Bodens als Ursachenfaktor auszuschließen.

Der Einsatz des Verfahrens im Rahmen sozialwissenschaftlicher Experimente besteht aus einer Verbindung des Parallelgruppenmodells (siehe oben) und der obigen Idee der Randomisierung. Die Gruppe der potenziellen VersuchsteilnehmerInnen wird dabei (vor der Einteilung in die Untersuchungsgruppen) hinsichtlich spezifischer Merkmale vorgruppiert. Es handelt sich dabei um Merkmale, die für die Erhebung als potenziell einflussreich betrachtet werden. Gängig ist hier beispielsweise eine Differenzierung nach Geschlecht, Alter oder Häufigkeit der Experimentteilnahme. Die Versuchspersonen aus diesen Schichten werden im Anschluss an diese Vorgruppierung mit Hilfe eines Zufallsverfahren der Kontrollund Versuchsgruppe zugeordnet. Wird das Geschlecht beispielsweise als ein potenziell relevanter Einflussfaktor angesehen, so wird durch die Vorgruppierung sichergestellt, dass jeder Untersuchungsgruppe gleich viele Männer und Frauen zugeordnet werden. Bei großen Stichproben wird angenommen, dass sich weitere unbekannte Störfaktoren auf der Grundlage dieses Vorgehens im Mittel ausgleichen und einer systematischen Verzerrung der Ergebnisse vorgebeugt werden kann. ${ }^{1}$ Es wird dabei der Annahme gefolgt, dass stets unkontrollierbare sowie unbekannte Störvariablen vorliegen, die das Ergebnis der experimentellen

\footnotetext{
${ }^{1} \mathrm{Zu}$ unterscheiden von der Randomisierung ist das Matching. Während die Störquellen mit dem Verfahren der Randomisierung durch das Design der Erhebung ausgeschlossen werden sollen, wird dies beim Verfahren des Matching durch die nachträgliche Analyse angestrebt. Nach der Datenerhebung erfolgt dabei eine nachträgliche Zuordnung von Versuchspersonen zu bestimmten Blöcken, die gemeinsame Merkmale aufweisen. Ziel ist es hier, die Wirkung dieser Merkmale auf die Ergebnisse in der Experimental- und Kontrollgruppe zu kontrollieren. Die zentrale Differenz zur Randomisierung ist jedoch, dass nur gemessen werden kann, was
} 
Erhebung ursächlich beeinflussen können, und dass diese Störvariablen in der untersuchten Population in unterschiedlichen Ausprägungen vorhanden sind. Die zufällige Zuweisung der ProbandInnen zur Kontroll- und Versuchsgruppe wird nun als Möglichkeit betrachtet, eine systematische und verzerrende Einflussnahme auf die Ergebnisse der Datenerhebung zu vermeiden. Dieser Anspruch wird damit begründet, dass durch die randomisierte Zuteilung zu den beiden Gruppen auch der Einfluss der Störvariablen auf symmetrische Weise gestreut wird und somit in den verschiedenen Untersuchungsgruppen auf die Ergebnisse in gleicher Weise einwirkt. Je größer die Stichprobe einer experimentellen Erhebung, desto wahrscheinlich ist es, dass sich die Einflüsse der Störvariablen auf die Datenerhebung ausgleichen. Die Idee des Verfahrens der Randomisierung besteht somit darin, den störenden Einflussfaktor auf der Ebene der spezifischen Erhebungssituation anzuerkennen und anzunehmen, dass sich dieser in den aggregierten Daten der einzelnen Erhebungen ausgleichen wird. Die Lösung des Problems des Einflusses der Störvariablen wird durch eine Verschiebung auf eine höhere Verallgemeinerungsebene angestrebt. Auf der Basis der Annahme, dass durch die Zufallszuweisung der ProbandInnen zu den Untersuchungsgruppen eine gleichmäßige Streuung der Störvariablen vorliegt, wird das Problem hier als analytisch beherrschbar betrachtet.

Standardisierung und Randomisierung bilden Verfahren, die im Experimentallabor gezielt eingesetzt werden, um den Anforderungen der Gleichartigkeit der Randbedingungen der Erhebung und der Wirkung des Stimulus auf die Versuchspersonen zu entsprechen. Die Art und Weise, wie Standardisierung und Randomisierung im Experimentallabor performativ erzeugt und genutzt werden, bilden einen wichtigen Aspekt der nachfolgenden empirischen Analyse, da sie für die Herausbildung der Experimenthaftigkeit der sozialen Veranstaltung von großer Bedeutung sind. Wie die empirische Analyse aufzeigen wird, wird der Mechanismus der Randomisierung auch innerhalb der Experimentsituation für die zufällige Zuweisung zu spezifischen Arbeitsplätzen im Labor verwendet. In dieser Variante zielt die zufällige Zuweisung unter anderem darauf $a b$, potenziell existente Gruppen einander bekannter ProbandInnen voneinander zu separieren, um diesen Aspekt als Störfaktor auszuschließen.

\section{Replikation}

Aufbauend auf dem Anspruch der Kontrolle der Erhebungssituation gilt die Wiederholbarkeit einer experimentellen Erhebung als ein anzustrebendes Ideal.

auch bekannt ist (Berger \& Wolbring 2015: 42). Der Einfluss unbekannter Störvariablen ist somit nicht kalkulier bzw. ausschaltbar. 
Experimente und ihre Ergebnisse sollen auch von anderen Forschenden (in ihren Laboren) wiederholt werden können und die gleichen Ergebnisse hervorbringen. Die wiederholte Erhebung mittels einer Versuchsanordnung wird allgemein als Replikation bezeichnet. Mit der Kontrolle der Untersuchungsbedingungen und dem Konstanthalten der Randbedingungen im Rahmen eines Experiments geht zugleich der Anspruch einer Wiederhol- oder Replizierbarkeit der Versuche einher. Es werden dabei die gleichen Hypothesen mit gleichen Methoden geprüft. Die Replikation kann dabei in einem anderen Labor als dem der ursprünglichen Erhebung erfolgen. Werden in demselben Labor wiederholt Erhebungen der gleichen Versuchsanordnung durchgeführt, so können diese als (direkte) Replikation bezeichnet werden. Allerdings werden andere Stichproben aus der Grundgesamtheit der verfügbaren Versuchspersonen gezogen und so neue Daten generiert.

Wenn in wiederholten Durchführungen gezeigt werden kann, dass sich die Ergebnisse aus unterschiedlichen Stichproben in signifikantem Maße gleichen, so wird angenommen, dass ein systematischer Zusammenhang zwischen den hypothesenrelevanten Variablen vorliegt. Berger (2015: 71) differenziert noch einmal zwischen Reproduktion und Replikation. Reproduktion bezieht sich dabei auf die erneute Herstellung von Daten als Ergebnis, wohingegen die Replikation die Wiederholung eines Versuchsaufbaus bezeichnet. Die Replikation von einer Versuchsanordnung wird jedoch nicht nur zur Absicherung der eigenen Ergebnisse eingesetzt. Häufig bildet eine Replikation eines bekannten und anerkannten Experiments den Ausgangspunkt nachfolgender experimenteller Erhebungen. Diese bauen argumentativ auf den als bewiesen erachteten Ergebnissen auf und versuchen, diese in einem ersten Schritt selbst zu replizieren. Mit einer erfolgreichen Replikation (und Reproduktion) ${ }^{2}$ eines Experiments wird zugleich an den Validitätsanspruch dieses Experiments hinsichtlich des interessierenden Kausalzusammenhangs angeschlossen. Eine erfolgreiche Replikation bekannter Experimente oder bestehender Forschungsergebnisse stellt im Fall wirtschaftswissenschaftlicher Experimente häufig den Ausgangspunkt für die eigene Erhebung einer Versuchsserie dar. Diese grundlegende experimentelle Versuchsanordnung wird

\footnotetext{
${ }^{2}$ Im Sinne eines Feldbegriffes wurde von den untersuchten Experimentalforschenden stets von Replikation gesprochen, auch wenn das Ziel eine Reproduktion von Daten darstellte. Eine mögliche Erklärung könnte jedoch auch in der Popularität des Begriffs ,replication“ und ,replication studies “ in der englischsprachigen Diskussion der Experimentalforschenden liegen.
} 
als ,Baseline Treatment ' bezeichnet. ${ }^{3}$ Nachfolgend werden durch gezielte Erweiterungen und Veränderungen der Versuchsanordnung neue Hypothesen getestet. Darauf aufbauend werden weitere experimentelle Anordnungen konzipiert, die sich in (einer) relevanten Dimensionen von dem Baseline Treatment unterscheiden. Von zentraler Relevanz ist es dabei, die ursprünglichen Experimente möglichst genau zu replizieren, damit das Ergebnis der nachfolgenden Veränderung der Versuchsanordnung auf den manipulierten Einflussfaktor zurückgeführt werden kann.

\section{Interne und externe Validität}

Die Güte der Ergebnisse eines experimentellen Forschungsdesgins wird mittels zweier zentraler Aspekte bestimmt: der internen Validität und der externen Validität (Campbell \& Stanley 1963). Hinsichtlich der Diskussion der Spezifik von Laborexperimenten sind diese Gütekriterien insofern von Belang, als dass den Laborexperimenten im Vergleich zu den anderen Formen experimenteller Forschungsdesigns ein besonders hohes $\mathrm{Ma} ß$ an interner Validität zugesprochen wird, jedoch nur ein geringes Maß an externer Validität (Kromrey 2002: $501 \mathrm{f}$.). Die interne Validität ist das Kriterium für den Anspruch, dass die Ausprägung der abhängigen Variablen das Resultat der Variation der unabhängigen Variablen ist (Campell 1957; Krebs \& Menold 2014: 436). Für den angenommenen Kausalzusammenhang zwischen den Variablen darf es also keine zulässigen Alternativerklärungen geben. Das Untersuchungsdesign des Experiments ist in seinem Ideal auf die Maximierung der internen Validität ausgerichtet, denn sein Ziel besteht ja gerade darin, durch Kontrolle, Isolation, das Konstanthalten der Rahmenbedingungen und die Minimierung der Wirkung potenzieller Störvariablen ein optimales Untersuchungsumfeld für eben diese Kausalanalyse herzustellen. Jede erfolgreiche Durchführung und Replikation mit einer gleichen Versuchsanordnung und einer anderen Stichprobe trägt dabei zur internen Validität der Ergebnisse bei.

Die externe Validität einer Studie bezieht sich hingegen auf die Verallgemeinerbarkeit und Generalisierbarkeit der Ergebnisse. Dieses Gütekriterium wird daran gemessen, inwiefern die Ergebnisse eine Robustheit gegenüber von Veränderungen der Versuchssituation und sich ändernden Stichproben aufweisen (Zimmermann 2015: 18). Lässt sich der unterstellte Kausalzusammenhang auch

\footnotetext{
${ }^{3}$ Es handelt sich dabei im Sinne der Grundlagen sozialwissenschaftlicher Experimente um die Kontrollgruppe.
} 
in diesen Fällen valide nachweisen, so erhöht sich die externe Validität der experimentellen Studie. Jede erfolgreiche Reproduktion ${ }^{4}$ der Ergebnisse einer Studie, in der die Untersuchungsbedingungen leicht verändert wurden, führt dabei zu einer Steigerung der externen Validität. Die Ergebnisse weisen in diesen Fällen eine gewisse Robustheit gegenüber den Veränderungen der Untersuchungsbedingungen auf. Ein Aspekt externer Validität ist die formale Frage, ob die Stichprobe der Versuchspersonen auch repräsentativ für die Grundgesamtheit ist, über die mit der Forschungsfrage eine Aussage getroffen werden soll. Ein hohes Maß an externer Validität liegt vor, wenn die relevanten Merkmale der Grundgesamtheit gleichsam auch in der untersuchten Stichprobe vorhanden sind (Krebs \& Menold 2014: 435). Andererseits ist der Anspruch der Generalisierbarkeit mit der Forderung verbunden, dass die Untersuchungsanordnung auch den Kern der interessierenden Wirkbeziehungen in der sozialen Wirklichkeit abbilden muss. Zimmermann (2015: 18) verweist darauf, dass externe Validität nicht danach zu bestimmen ist, ob Experimente ein getreues Abbild der sozialen Realität erzeugen, sondern dass entscheidend ist, dass sie die zentralen Einflussfaktoren und ihre Folgen erfassen. Der intendierte Untersuchungsgegenstand muss somit mit dem übereinstimmen, der in der experimentellen Versuchsanordnung erzeugt und gemessen wurde. Dieser Aspekt der Verallgemeinerbarkeit wird häufig auch als „ökologische Validität“ bezeichnet (Zimmermann 2015: 18 f.). Je reduzierter und künstlicher die Erhebungssituation ist, desto größer ist dabei die Gefahr, die komplexen Zusammenhänge der sozialen Wirklichkeit zu Unrecht zu verkürzen. Je natürlicher die Situation der Erhebung hinsichtlich der Komplexität der Zusammenhänge ist, desto höher ist dementsprechend die externe Validität. Interne Validität und die externe Validität im Sinne der Verallgemeinerbarkeit stehen in einem gewissen Spannungsverhältnis zueinander. Diesem tragen die unterschiedlichen Varianten der experimentellen Designs Rechnung.

\section{Varianten des experimentellen Designs in der Sozialwissenschaft}

Da sich meine folgende Analyse der Untersuchung von Laborexperimenten widmet, soll diese Variante des experimentellen Forschungsdesigns kurz von zwei anderen Formen abgegrenzt werden. Die Unterscheidung erfolgt hier nur bezogen auf den idealtypischen wissenschaftlichen Anspruch, der mit diesen Varianten experimentellen Designs verbunden wird, und nicht auf der Grundlage einer

\footnotetext{
${ }^{4}$ Berger (2015) verwendet an dieser Stelle den Begriff der „Reproduktion“ statt Replikation. Diese Differenzierung erscheint für die Wiederholung von Studien zur Überprüfung der externen Validität als aufschlussreich. Primäres Ziel ist ja hier, die Ergebnisse erneut zu generieren und nicht die Versuchsanordnung nachzustellen. Reproduktion kann also eine Replikation beinhalten, muss dies aber nicht.
} 
detaillierten empirischen Analyse. Er dient der Eingrenzung der Variante des experimentellen Designs, die Gegenstand dieser Arbeit ist.

Dem Idealtypus des experimentellen Designs kommt das Laborexperiment am nächsten. Dieses Forschungsdesign bietet aus Sicht der Forschenden die besten Möglichkeiten, den Anspruch an Kontrolle und Isolation der hypothesenrelevanten Variablen zu realisieren. Es wird angenommen, dass der Ablauf des Experiments hier in entscheidendem Maße von den Forschenden kontrolliert werden kann, wodurch der Einfluss von Störvariablen stark reduziert wird. Aufgrund der Künstlichkeit der Erhebungssituation gelten Laborexperimente als besonderes geeignet, um die Komplexität der sozialen Wirklichkeit zu reduzieren und hypothesenrelevante Variablen gegenüber störenden Einflüssen abzuschirmen (Wolbring \& Keuschnigg 2014: 119). Die interne Validität wird aus diesem Grund als besonders hoch betrachtet. Die vereinfachende Reduktion realweltlicher Komplexität der untersuchten Phänomene wird jedoch zugleich als deutliche Beschränkung der Verallgemeinerbarkeit der Ergebnisse angesehen und somit auch als Grund für eine Reduktion und Beschränkung der externen Validität der Untersuchungen.

Dem Problem der geringen externen Validität begegnen Feldexperimente mit einer spezifischen Abwandlung des Forschungsdesigns (ebd.: 220). Auch hier werden zu Beginn Hypothesen über den Kausalzusammenhang von abhängigen und unabhängigen Variablen aufgestellt. Die unabhängige Variable wird im Folgenden gezielt manipuliert. Die Wirkung auf die abhängige Variable wird hingegen in ihrem natürlichen Umfeld untersucht. Da die Manipulation des Einflussfaktors gezielt eingesetzt wird, können Kontroll- und Versuchsgruppen voneinander unterschieden werden. Durch das Parallelgruppenmodell ist es möglich, dass Verfahren der Randomisierung bei der Stichprobenauswahl zu nutzen, um die Wirkung von Störvariablen zu reduzieren. Der weitere Ablauf der Erhebung kann im Unterschied zum Laborexperiment in diesem Fall jedoch nicht vor der Wirkung von Störvariablen abgeschirmt werden.

Zusammenfassend zeichnen sich Feldexperimente dadurch aus, dass sie einerseits über die Grundelemente des experimentellen Forschungsdesigns (Manipulation der untersuchten Variablen, Parallelgruppenmodell und Randomisierung) verfügen, zugleich jedoch in einem natürlichen Umfeld durchgeführt werden (ebd.: 224). Nach dem manipulativen Eingriff ist daher die Kontrolle durch den Forschenden stark begrenzt und die Ceteris-Paribus-Klausel nur eingeschränkt gültig. Dies führt zu einer starken Beschränkung der internen Validität der Ergebnisse. Der unterstellte Kausalzusammenhang kann somit nicht zweifelsfrei nachgewiesen werden, da nie ausgeschlossen werden kann, dass Störvariablen 
einen maßgeblichen Einfluss auf das Ergebnis ausgeübt haben. Die externe Validität der Ergebnisse ist im Vergleich zum Laborexperiment jedoch deutlich größer. Eine Differenz zwischen den Untersuchungsbedingungen der experimentellen Studie und den realweltlichen Zusammenhängen liegt kaum mehr vor.

Natürliche Experimente basieren auch auf einem Vergleich von Versuchs- und Kontrollgruppe. Die Differenz zwischen beiden Gruppen wird hier jedoch nicht aktiv durch den Eingriff des Forschenden erzeugt, sondern durch eine nachträgliche Zuordnung. Es handelt sich vielmehr um eine nachträgliche Analyse von unterschiedlichen Phänomenen, die sich in vielen zentralen Merkmalen gleichen, jedoch hinsichtlich eines Merkmals unterscheiden (Bauer 2015). ${ }^{5}$ Es besteht hier keine Möglichkeit des Konstanthaltens der Untersuchungsbedingungen. Die Randomisierung stellt ein zentrales Definitionsmerkmal experimenteller Studien dar, die auf valide Kausalanalyse abzielen, und bildet zugleich eine grundlegende Differenz zwischen dieser dritten Form des experimentellen Designs und den beiden erstgenannten. Während die Zuweisung zu Versuchs- und Kontrollgruppen in Feld- und Laborexperimenten durch die Forschenden erfolgt, geschieht dies in natürlichen Experimenten durch die Natur selbst. Die Qualität der Ergebnisse hinsichtlich des untersuchten Kausalzusammenhangs hängt somit maßgeblich davon ab, ob tatsächlich angenommen werden kann, dass die Zuweisung zu den Untersuchungsgruppen zufällig erfolgte (ebd.: 270). Auch der Einfluss von Störvariablen kann im Rahmen eines natürlichen Experiments nicht kontrolliert werden. Die interne Validität wird somit stark herabgesetzt. Die externe Validität wird wegen der Natürlichkeit des Erhebungszusammenhangs zugleich jedoch maximal erhöht. Auch wenn mittlerweile wohl alle drei oben genannten Formen von experimentellen Forschungsdesigns im Bereich der Wirtschaftswissenschaften angewendet werden (Kübler 2014), verweist der Begriff der experimentellen Wirtschaftsforschung zumeist auf eine spezifische Form des Experiments, welches zur Datenerhebung genutzt wird. Die typische Variante des experimentellen Forschungsdesigns im Bereich der Ökonomie bilden die nachfolgend thematisierten verhaltensökonomischen Laborexperimente.

\footnotetext{
${ }^{5}$ Ein Beispiel wäre die Einführung des bedingungslosen Grundeinkommens in Finnland und ein Vergleich mit einem anderen skandinavischen Land. Im Folgenden könnte nun beispielsweise ein Vergleich hinsichtlich bestimmter Gesundheits- oder Bildungsfragen unternommen werden, wie beispielsweise ob zwei Jahre nach Einführung signifikant mehr Menschen ein Studium begonnen oder weniger Personen eine Behandlung wegen psychischer Erkrankungen wie Depressionen und Burn-Out-Syndromen begonnen haben.
} 


\subsection{Grundmomente der experimentalökonomischen Forschungsperspektive}

Nach dieser grundlegenden Einführung von zentralen Annahmen des experimentellen Forschungsdesigns in den Sozialwissenschaften widmet sich der folgende Abschnitt dem Feld der experimentellen Wirtschaftswissenschaft. Ziel der Ausführungen ist es, ein Verständnis für die Perspektive der Forschenden $\mathrm{zu}$ ermöglichen, welche Laborexperimente in ihrer wirtschaftswissenschaftlichen Forschung einsetzen. Dabei besteht ein erster Schritt darin aufzuzeigen, dass durch die mathematisch-formalisierende Darstellung ökonomischer Phänomene der Grundstein zur Etablierung der experimentellen Methode gelegt wurde. Die Mathematisierung der Ökonomie bildet deshalb den argumentativen Ausgangspunkt der Darstellung (Abschnitt 2.2.1). Im Anschluss wird mittels eines kurzen historischen Abrisses die Etablierung des experimentellen Vorgehens im Bereich der Wirtschaftswissenschaft nachgezeichnet (Abschnitt 2.2.2). In einem abschließenden Schritt wird in dieser thematischen Einführung dargestellt, welche Erwartungen mit der Anwendung von wirtschaftswissenschaftlichen Experimenten (Abschnitt 2.2.3) verbunden werden.

\subsubsection{Mathematisierung der Ökonomie und die Spieltheorie}

Die Etablierung der Spieltheorie und die Mathematisierung der Ökonomie bilden zentrale Grundlagen für die Verbreitung des experimentellen Untersuchungsdesigns in der Wirtschaftswissenschaft. Aus diesem Grund wird dieser Zusammenhang kurz ausgeführt, um diesem wissenschaftssoziologisch relevanten Aspekt Rechnung zu tragen. Hinsichtlich des Einsatzes von Experimenten in der ökonomischen Forschung und dem Aufstieg der Spieltheorie besteht eine gewisse zeitliche Parallelisierung. Auch die Nutzung von Experimenten wurde lange Zeit vom wissenschaftlichen Mainstream der ökonomischen ForscherInnen als exotischer Sonderweg betrachtet. Wie die Ausführungen zeigen werden, etablierte sich die Spieltheorie anfangs nur zögerlich als wissenschaftliche Methode im Bereich der ökonomischen Forschung. Das zentrale Problem bestand in der für die damalige Zeit ungewöhnlich formalisierenden und mathematischen Darstellung ökonomischer Phänomene.

Die Mathematisierung der ökonomischen Disziplin war zu Beginn des 20. Jahrhunderts noch wenig fortgeschritten. Weintraub „How Economics Became a Mathematical Science“ (2002) liefert einen geschichtlichen Abriss der Hintergründe dieser Entwicklung. Etwa zeitgleich mit dem Aufkommen der Spieltheorie 
vollzog sich ein deutlicher Wandel in der Ökonomie: Langsam aber stetig erfolgte eine zunehmende Orientierung der wirtschaftswissenschaftlichen Forschung am Ideal der naturwissenschaftlichen Disziplinen, allen voran an der Physik. ${ }^{6}$ Auch in der Ökonomie setze sich zunehmend die Ansicht durch, dass sich die für die Disziplin relevanten Phänomene auch durch die formale Sprache der Mathematik darstellen lassen würden. Samuelson (1952) argumentiert für eine gleichrangige Betrachtung deskriptiver und mathematischer Phänomenbeschreibungen, da ökonomische Fragestellungen ihrer Natur nach quantitativ seien: „,[P]roblems of economic theory $[\ldots]$ are by their nature quantitative questions whose answer depends upon a superposition of many different pieces of quantitative and qualitative information. When we tackle them by words, we are solving the same equations as when we write out those equations" (Samuelson 1952: $63 \mathrm{f}$.).

Die mathematische Schreibweise wurde dabei allerdings von Samuelson noch nicht als ein Weg gesehen, die qualitativen Dimensionen der beobachteten Phänomene auszuschalten: "I wish I thought it were true that the language of mathematics had some special faculty of drawing attention away from the pseudo problems of qualitative essence [...] I should consider that a great advantage" (ebd.: 63). Diese Erhöhung des Abstraktionsgrades, der sich lediglich der Betrachtung quantitativer (und zugleich klar mathematisch formalisierbarer) Dimensionen zuwandte, war in dieser Phase der Entwicklung der ökonomischen Disziplin noch nicht verbreitet. Die historisch deskriptive Darstellung und Analyse ökonomischer Problemstellungen entsprach zu dieser Zeit dem gängigen Vorgehen. Der deutsche Mathematiker und Ökonom von Stackelberg fasste $1943^{7}$ die Kritik und zugleich den Vorteil der Anwendung der Mathematik wie folgt: „[Es] wird eingewendet, die Mathematik täusche eine Exaktheit und Starrheit der volkswirtschaftlichen Beziehungen vor, die in Wirklichkeit fließend und unexakt seien; sie täusche naturgesetzliche Notwendigkeit vor, wo in Wirklichkeit der menschliche Wille frei entscheiden und gestalten könne. Deshalb sei die Anwendung der Mathematik in der Volkswirtschaftslehre abzulehnen“" (von Stackelberg 1951: XII).

\footnotetext{
${ }^{6}$ Einer Darstellung der Übertragung physikalischer Prinzipien auf den Bereich der Ökonomie widmet sich Mirowski (1989) in „More Heat than Light. Economics as Social Physics, Physics as Nature"s Economics". Mirowski zeichnet hier nach, wie die Verwendung von Metaphern aus dem Bereich der Physik in der Ökonomie zur Entwicklung und dem Wandel zentraler theoretischer Positionen führten. Einen zentralen Fokus bilden dabei die Erkenntnisse, die in der Physik durch den Energieerhaltungssatz begründet und in der Ökonomie seit dem späten 19. Jahrhundert aufgenommen und verarbeitet wurden. (Corry 2003: 289).

${ }^{7}$ Hinsichtlich der zeitlichen Verortung sei darauf verwiesen, dass das hier zitierte Textfragment der zweiten Auflage von Stackelberg „Grundlagen der theoretischen Volkswirtschaftslehre“ (1951) entstammt. Gewidmet und verfasst war dieses jedoch als Vorwort der ersten Auflage, welche bereits 1943 erschien.
} 
Auch von Stackelberg nahm hier Bezug auf die gängige Kritik der historisch und deskriptiv ausgerichteten Theoretiker. Diese Kritik bestand darin, dass die Komplexität und die qualitativen Dimensionen und Beziehungen, die den betrachteten wirtschaftlichen Phänomenen zugrunde liegen, in mathematischen Darstellungen verzerrt dargestellt und ungenügend repräsentiert wären. Doch von Stackelberg warf diesen Kritikern vor, die Rolle der Mathematik in der Wirtschaftstheorie falsch einzuschätzen. Er verwies aus diesem Grund darauf, dass ,aus dem mathematischen Topf nie mehr herausspringt, als in ihn vorher hineingelegt worden ist" (von Stackelberg 1951: XII). Die mathematische Symbolik würde weder die Voraussetzungen noch die Folgerungen eines Theoretikers verändern und beeinflussen, solange diese selbst schlüssig seien.

„Was die Mathematik allein bewirkt, ist ein genaues Denken, auch über, ungenaue Dinge'; und das ist allerdings sehr viel. Teilweise ist es in unserem Fach Mode, in Anbetracht der großen Kompliziertheit des Gegenstandes mit großem Schwung über die Schwierigkeiten hinwegzugleiten und aus der Fülle anschaulicher und unanschaulicher Phantasie umfangreiche Gedankengebilde zu formen. Das wird allerdings durch die Mathematik vollständig unmöglich gemacht. Die Mathematik zwingt vielmehr das Denken zu unerbittlicher Selbstdisziplin. “ (ebd.)

Für von Stackelberg veränderte die mathematische Darstellung nicht die Inhalte, die durch sie dargestellt wurden. Er betrachtete die Übertragung ökonomischer Erklärungsansätze in mathematische Formeln als eine reine ,Übersetzung ${ }^{c}$, durch welche deskriptive Beschreibungen bereichert und ergänzt werden sollten. Den großen Vorteil mathematischer Modelle sahen die ökonomischen ForscherInnen in der Nachvollzieh- und Beweisbarkeit der Logik von Theorien, Gleichungen und Modellen und in der damit einhergehenden Offenlegung von Annahmen und Prinzipien. Man war bemüht, die ökonomischen Theorien auf evidenten Prämissen zu gründen, welche ihre Geltung auch ohne empirische Verifikation behaupten konnten (Jonas 1964: 96). Zur empirischen Überprüfung beschränkte man sich auf Methoden der vergleichenden Statistik und Ökonometrie (Dos Santos 2010: 82).

Hinsichtlich der Entwicklung der ökonomischen Perspektive und Gegenstandskonzeptualisierung zeigt sich jedoch, dass von Stackelberg die Konsequenzen der Mathematisierung für die eigene Disziplin unterschätzte. Die zunehmende Verwendung der mathematischen Formalisierung zur Beschreibung ökonomischer Probleme war nicht, wie von Stackelberg darlegte, auf eine inhaltliche Übersetzung von Worten in mathematische Zeichen beschränkt. Vielmehr ging mit dieser Übertragung auch die Aufnahme der strukturellen Logik der mathematischen Formalisierung einher. Aus wissenssoziologischer Perspektive ließe sich 
dies mit Verweis auf Simmel (1908) dadurch begründen, dass durch die Quantifizierung der Phänomene sowohl ,Inhalt' als auch ,Form' der ökonomischen Problemstellungen und -analysen verändert wurden. Von Stackelbergs Argumentation, dass ,aus dem mathematischen Topf nie mehr herausspringt, als in ihn vorher hineingelegt worden ist" übersah somit, dass die mathematische Formalisierung zu einer anderen Form und Logik der wissenschaftlichen Argumentation führte.

\section{Die Spieltheorie als wissenschaftliche Beschreibungssprache von Entscheidun- gen}

Wie oben angemerkt, zeigt sich eine Parallelentwicklung zwischen der Mathematisierung der Ökonomie und der Etablierung der Spieltheorie als formaler Sprache der Beschreibung von Entscheidungssituationen. Der nachfolgende kurze historische Abriss über die Begründung der Spieltheorie zielt darauf $a b$, einige dieser Prozesse kurz darzustellen und in diesem Rahmen einige Grundgedanken der Spieltheorie einzuführen, welche sich in der späteren Diskussion von Experimenten als wirtschaftswissenschaftlichem Forschungsdesgin und ihrer -praxis als bedeutsam erweisen werden.

Als Begründer der Spieltheorie gilt der ungarische Mathematiker John von Neumann. In seinem Aufsatz „Zur Theorie der Gesellschaftsspiele“ (1928) stellte von Neumann fest, dass es eine grundlegende Ähnlichkeit zwischen den Verhaltens- und Entscheidungsstrategien von Menschen bei Schach- und Gesellschaftsspielen und anderen sozialen Situationen gibt, in welchen die Handelnden miteinander in Konkurrenz treten. Diese grundlegende Analogie zu Gesellschaftsspielen ist es auch, von der sich der Name ,Spieltheorie" ableitet. Von Neumann widmet sich der Frage, wie Spieler ${ }^{8}$ spielen müssen, um ein möglichst günstiges Ergebnis zu erzielen (vgl. ebd.: 295). ${ }^{9}$ Die zentrale Grundlage

\footnotetext{
${ }^{8}$ In der nachfolgenden Darstellung der Grundlagen der Spieltheorie werden die Begriffe „Entscheider“, „Spieler“, „Mitspieler“ und „Gegenspieler“ nicht gegendert, da diese hier im Sinne der Theoriesprache der Spieltheorie verwendet werden, in welcher nur die maskuline Form der Wörter Verwendung findet.

${ }^{9}$ Bereits weit vor von Neumann gab es andere Autoren, die sich der mathematischen Darstellung von Spielsituationen widmeten. Der erste Versuch geht wohl auf das Jahr 1713 zurück, in welchem sich Francis Waldegrave, Pierre-Rémond de Montmort und Nicolas Bernoulli in mehreren Briefwechseln über unterschiedliche Lösungskonzepte für das zwei-Personen-Kartenspiel austauschten (siehe auch Hykšová 2004: 50). Eine ausführliche wirtschaftshistorische Auseinandersetzung der Entstehungsgeschichte der Spieltheorie sowie mit der Biographie von Neumanns und Morgensterns bietet zudem das Werk von Robert J. Leonard (2010) ,Von Neumann, Morgenstern, and the Creation of Game Theory: From Chess to Social Science, 1900-1960“.
} 
der Spieltheorie ist dabei die Beobachtung, dass es den Spielern nicht möglich ist, die Konsequenzen ihres Spielzuges eindeutig zu bestimmen, da sie keine Kontrolle darüber besitzen, wie ihr ,Gegenspieler' auf ihren Zug reagieren wird. Vor der Entscheidung für einen eigenen Zug ist es somit nötig zu antizipieren, welche Reaktion der eigene Zug wohl beim Gegenspieler auslösen wird und welche Reaktion die Gegenspieler wiederum auf ihrem Zug erwarten. Eine Spielsituation bildet sich für von Neumann, sobald mehrere Personen zusammenkommen und miteinander interagieren. Denn in diesen Fällen hängt nach von Neumann „das Schicksal eines jeden Spielers außer von seinen eigenen Handlungen auch noch von denen seiner Mitspieler ab; und deren Benehmen ist von genau denselben egoistischen Motiven beherrscht, die wir beim ersten Spieler bestimmen möchten. Man fühlt, daß ein gewisser Zirkel im Wesen der Sache liegt“ (ebd.).

Gegenstand der Spieltheorie sind somit Situationen, in denen das Ergebnis nicht nur von den eigenen Entscheidungen einer Person abhängt, sondern auch vom Verhalten anderer Spieler. Solch eine Entscheidungssituation, in der mehrere vernunftbegabte Entscheider ihre eigenen egoistischen Interessen verfolgen, wird aus spieltheoretischer Perspektive als strategischer Konflikt, strategische Interaktion oder strategisches Spiel bezeichnet (Rieck 1993: 16). Bei der grundlegenden Betrachtung spieltheoretischer Entscheidungssituationen wird somit von einem rational handelnden Akteur ausgegangen, der durch die Wahl entsprechender Entscheidungs- bzw. Handlungsalternativen bemüht ist, seinen erwarteten Nutzen zu maximieren (Holler \& Illing 1996: 10).

Die beiden Ökonomen Manfred Holler und Gerhard Illing (1996) charakterisieren die strategischen Entscheidungssituationen, die den Gegenstand der Spieltheorie bilden, als Situationen, die vier Charakteristika erfüllen:

1. Das Ergebnis dieser Situationen hängt von den Entscheidungen mehrerer Entscheidungsträger $\mathrm{ab}$.

2. Jeder beteiligte Entscheidungsträger ist sich dieser Interdependenz bewusst.

3. Jeder Entscheidungsträger geht davon aus, dass sich alle anderen ebenfalls der Interdependenz bewusst sind.

4. Jeder Entscheider berïcksichtigt bei seiner Entscheidung Punkt 1, 2 und 3. (vgl. ebd.: 1)

Hier zeigt sich der zentrale Unterschied zur klassischen Entscheidungstheorie, in der ausschließlich Situationen betrachtet werden, in denen gegen die Natur gespielt wird und damit die Wahrscheinlichkeitsverteilungen über Umweltzustände vom eigenen Verhalten der Entscheider als unabhängig betrachtet werden (Davis 2005: 16). Ihrem Selbstverständnis nach ist die Spieltheorie damit eine 
,Theorie sozialer Interaktion" und wird auch als ,interaktive Entscheidungstheorie' (Rieck 1993: 16) bezeichnet. Grundlegend für die spieltheoretische Betrachtung von strategischen Interaktionssituationen ist, dass diese in einer Spielform dargestellt werden. Die einzelnen Personen werden dabei zu Spielern oder Agenten, die auf die Maximierung ihres eigenen Nutzens abzielen und dadurch geleitet bestimmte Handlungsstrategien im Zuge der Interaktion auswählen. Die einzelnen Entscheidungen der Spieler werden dabei als ,Züge" bezeichnet. Spielsituationen beschränken sich dabei häufig nicht auf eine einzige, isolierbare Entscheidung (wie beispielsweise beim Gefangenendilemma ${ }^{10}$ ), sondern sind komplex und bestehen aus mehreren miteinander verbundenen Entscheidungen.

Wie im Zuge des Theoriekapitels (3.1) ausgeführt wird, basiert auch das Rahmenkonzept Goffmans maßgeblich auf seinen Arbeiten zur strategischen Interaktion, welche zentral auf der Analogie zur Spieltheorie und zum Spielen im Allgemeinen fußt. Die Goffmansche Perspektive auf das strategische Spiel erweist sich für die nachfolgende Analyse als fruchtbarer Anknüpfungspunkt, um die spezifischen Rahmenbildungsprozesse und die Modulation der Alltagssituation zu betrachten, die im ökonomischen Laboratop erzeugt wird. In der Differenz von Spieltheorie und der klassischen Entscheidungstheorie lässt sich zudem eine interessante Parallele zu Goffmans Unterscheidung von natürlichen und sozialen Rahmen entdecken, die ja auch maßgeblich auf der wahrgenommenen Unterscheidung ihrer natürlichen und sozialen (interaktiven) Hervorbringung gründet (Goffman, 1977: 36). Goffman betrachtet diese Unterscheidung zwischen natürlichen und sozialen Rahmen allerdings nicht als eine gegebene, sondern als sozial konstruierte Differenz (Willems 1997: 56).

\section{Der Eingang der Spieltheorie in die ökonomische Forschung}

Bereits auf der ersten Seite seines Aufsatzes von 1928 verweist von Neumann darauf, dass die von ihm thematisierte Problemstellung und die damit zusammenhängende Zirkularität des Entscheidungsverhaltens zugleich das Hauptproblem

\footnotetext{
${ }^{10}$ Das Gefangenendilemma bezeichnet eines der bekanntesten Spiele der Spieltheorie. Es handelt sich um eine Spielsituation, in der zwei Gefangene eines gemeinsamen Verbrechens beschuldigt werden. Ihre möglichen Züge bestehen darin, das Verbrechen entweder zu gestehen oder es zu leugnen. Leugnen beide das Verbrechen, erhalten sie nur eine geringe Strafe. Gestehen beide, ist ihre Strafe deutlich höher. Gesteht jedoch nur einer der beiden, geht der Geständige straffrei aus, wohingegen den anderen Gefangenen die Höchststrafe erwartet. Beide Gefangenen werden über die Konsequenzen ihre Entscheidungen informiert und nun einzeln befragt, können jedoch nicht miteinander kommunizieren und demzufolge keine Absprachen über eine gemeinsame Strategie tätigen (Davis 2005: 104 ff.).
} 
der klassischen Nationalökonomie darstellt: „,[W]as wird unter gegebenen äußeren Umständen, der absolut egoistische ,homo oeconomicus ‘ tun?“" (von Neumann 1928: 1). ${ }^{11}$ Der wissenschaftliche Durchbruch der Spieltheorie basiert 1944 auf der Veröffentlichung des Buches „Theory of games and economic behavior“ $(\text { TGEB })^{12}$, welches von Neumann zusammen mit dem Wirtschaftswissenschaftler Oskar Morgenstern verfasste. Die Autoren schildern hier anhand von vielfältigen Beispielen, wie ökonomische Fragen mittels mathematischer, formaler Modelle strategischer Spiele beschrieben und damit zugleich analysiert werden können (vgl. Morgenstern 2005 [1972]: 7; von Neumann \& Morgenstern 2007 [1944]: 1). Bereits auf der ersten Seite ihres Buches legen von Neumann und Morgenstern (2007) ihr Anliegen deutlich dar und verweisen darauf, dass sie auf die praktische Anwendung ihrer Theorie für den Bereich der Ökonomie abzielen:

„The purpose of this book is to present a discussion of some fundamental questions of economic theory which require a treatment different from that which they have found thus far in the literature. The analysis is concerned with some basic problems arising from a study of economic behavior which have been the center of attention of economists for a long time. They have their origin in the attempts to find an exact description of the endavor of the individual to obtain a maximum of utility, or, in the case of the entrepreneur, a maximum of profit. It will be made clear that the structure of the problems [...] is in many respects quite different from the way in which they are conceived at the present time. It will appear, furthermore, that their exact positioning and subsequent solution can only be achieved with the aid of mathematical method." (ebd.: 1)

Die Mathematik wird hier als eine Sprache der Logik verwendet, um die ökonomischen Problem- und Fragestellungen der Realität einer Analyse zugänglich zu machen. Die oben skizzierten grundlegenden Annahmen der Spieltheorie werden

\footnotetext{
${ }^{11}$ Das inhaltliche Ergebnis dieses Aufsatzes besteht im Beweis des Minimax-Theorems (oder auch Min-Max-Theorem) als einer fundamentalen Grundlage, auf der weitere spieltheoretische Arbeiten aufbauten (Morgenstern 1972: 7; Davis 2005: 45). Das Minimax-Theorem besagt, dass in einem Zweipersonenspiel-Nullsummenspiel (s. u.) rein mathematisch ein Wert als durchschnittlicher Gewinn bestimmt werden kann. Diese Idee folgt der mathematischlogischen Annahme, dass beide Spieler daran interessiert sind, die Gewinne des Gegenspielers zu minimieren und so zugleich ihre eigenen Gewinne zu maximieren. Gefolgt wird hierbei wieder der spieltheoretischen Annahme, dass es sich um Spieler handelt, die ihren eigenen Nutzen maximieren wollen und optimal spielen und somit keine Fehler in der Wahl ihrer Entscheidungsstrategie begehen.

${ }^{12}$ Die deutsche Veröffentlichung erfolgte 1961 unter dem Titel ,Spieltheorie und wirtschaftliches Verhalten“.
} 
dabei herangezogen, um die strategischen Konflikte und Interaktionen der beteiligten Spieler darzulegen und die aus ihnen hervorgehenden optimalen Lösungen aufzuzeigen. Ein zentrales Moment der Theorie von Neumann und Morgenstern ist die Annahme eines „egoistischen“ Akteurs nach dem Modell des Homo oeconomicus $^{13}$ (Machlup 1960: 42). Dieser Akteur besitzt eine feste Präferenzordnung und geleitet durch diese zielt er auf die Maximierung seines eigenen Nutzens ab. Die Rationalität der Handlungen eines Akteurs ist dabei an dieses Maximierungskalkül gebunden. Dieser Idee folgend, werden von den rationalen Akteuren stets die Entscheidungsalternativen oder Strategien gewählt, von denen sie den höchsten Nutzen erwarten. Es wird zugleich davon ausgegangen, dass auch die anderen Akteure diesem Ziel folgen. Die Lösung solcher strategischen Probleme lässt sich für von Neumann und Morgenstern mit Hilfe einer mathematischen Analyse der Spielsituationen bestimmen. Von Neumann und Morgenstern gingen dabei von solchen Situationen aus, in denen sich die Spieler in einem direkten Interessengegensatz zueinander befinden und so die Verluste des einen die Gewinne des anderen Spielers sind. Diese Situationen werden als ,Nullsummenspiel' bezeichnet (Davis 2005: 23).

Der Anspruch von Neumanns und Morgensterns bestand jedoch nicht nur darin, eine Inspiration oder Analogie für die Betrachtung ökonomischer Probleme aus spieltheoretischer Perspektive anzustoßen. „We hope to establish satisfactorily $[\ldots]$ that the typical problems of economic behavior become strictly identical with the mathematical notions of suitable games of strategy" (von Neumann \& Morgenstern 2007: 2) Vielmehr war es so, dass sie eine Gleichsetzung von ökonomischen Problemen und ihrer mathematischen Darstellung anstrebten. ${ }^{14}$

Viele Rezensionen namhafter Autoren in renommierten Fachzeitschriften, wie die des späteren Nobelpreisträgern Herbert A. Simon (1945 im „American Journal of Sociology“) und Richard Stone (1948 im „The Economic Journal“) erkannten das enorme Potenzial des Werkes von Neumanns und Morgensterns für die

\footnotetext{
${ }^{13}$ Zur einführenden Auseinandersetzung mit dem Modell des homo oeconomicus siehe Abschnitt 5.2.1.

${ }^{14}$ Weintraub (2002: 77 f.) verweist in diesem Zusammenhang darauf, dass von Neumann ein Schüler von Davis Hilbert war, welcher eine zentrale Figur in der Formalisierung der Mathematik darstellte. Hilberts Ideen hatten wiederum Einfluss auf die Arbeiten des ,Wiener Kreises', dessen Mitglied auch Morgenstern war. Der Wiener Kreis bezeichnet dabei einen Zirkel einflussreicher Wissenschaftler, die sich der empirischen Grundlegung der wissenschaftlichen Disziplinen verschrieben hatten. Zentrale Leidideen waren die Abkehr von der Metaphysik und die Hinwendung zu einem ,logischen Empirismus' (Verein Ernst Mach 2009). Die Einführung von von Neumanns und Morgensterns Spieltheorie in die Ökonomie bedingte damit zugleich, dass auch die Ideen der , stärkeren ' Formalisierung im Anschluss an Hilbert Aufnahme fanden.
} 
folgende Forschung. Wie Kuhn (2007) in seiner Einleitung zum Nachdruck des Werkes anlässlich des sechzigsten Jahrestags der TGEB darstellt, zeigte sich ein ,pattern of extravagant praise and no subsequent research“ (Kuhn 2007, IX). Trotz vielfältiger Lobpreisungen fand die Theorie von Neumanns und Morgensterns zu Beginn nur begrenzt Eingang in die ökonomische Forschung. Kuhn (2007) beschreibt, dass die mathematische Ausrichtung der Spieltheorie wohl der Grund für diese Probleme darstellte:

„A crucial fact was that von Neumann's theory was too mathematical for economists.
To illustrate the attitude of a typical economics department of the period and later,
more than fifteen years after the publication of TGEB the economists at Princeton voted
against instituting a mathematics requirement for undergraduate majors, choosing to
run two tracks of for students, one which used the calculus and one which avoided it
[...] Courses that used the mathematical terms and which covered mathematical topics
such as linear programming were concealed by titles such as "Marginal theory of the
firm." Given such views, there was no incentive or opportunity for graduate students
and junior faculty to study the theory of games. " (Kuhn 2007, xii)

Den Ausführungen Kuhns folgend lag ein weiterer Grund für die lang andauernde geringe Rezeption der TGEB zudem in der schwachen Institutionalisierung bzw. der Marginalisierung der statistisch-mathematischen Perspektive im Rahmen der wissenschaftlichen Ausbildung von jungen Ökonomen. Auch für die junge Generation von Forschenden wurden so wenig Anreize oder Berührungspunkte für eine Auseinandersetzung mit dem Potenzial dieses konzeptionellen Zugangs geschaffen. Die starke mathematische Orientierung der ökonomischen Forschung lag zu dieser Zeit noch nicht vor, sodass die Spieltheorie anfangs nur im Bereich der Mathematik angewendet und weiterentwickelt wurde.

Ein zentraler Fortschritt, der zur Etablierung der Spieltheorie beitrug, war der Beitrag von John Forbes Nash im Jahr 1950, der die Arbeiten von Neumanns und Morgensterns um das sogenannte ,Nash-Gleichgewicht" erweiterte. Das NashGleichgewicht beschreibt eine Lösung, bei der kein Spieler einen Vorteil dadurch erhält, einseitig seine Strategie zu ändern, wenn er davon ausgeht, dass die anderen Spieler ihre aktuelle Strategie beibehalten. Dieses Gleichgewicht stellt somit ein Lösungskonzept für Spiele mit mehr als zwei Spielern und auch für NichtNullsummenspiele bereit. Es zeigte sich jedoch bald, dass es in vielen Fällen nicht nur ein mögliches Nash-Gleichgewicht gibt. Eine in diesem Zusammenhang wichtige Weiterentwicklung des Nash-Gleichgewichtes ermöglichten die Arbeiten des deutschen Ökonomen Reinhard Selten, der nachwies, wie in Situationen mit mehreren Gleichgewichten eine dominierende Strategie bzw. ein dominierendes Gleichgewicht ermittelt werden konnte. John Harsanyi entwickelte Nashs 
Ansatz in der Hinsicht weiter, dass er mathematisch-spieltheoretisch ergründete, wie Spieler mit Informationsdefiziten umgehen, welche dazu führen, dass sie die Gegenzüge ihrer Mitspieler nur unvollständig kalkulieren können. Die Arbeiten von Selten und Harsanyi trugen maßgeblich zur Ausdifferenzierung der Spieltheorie bei und wurden, gemeinsam mit Nash, 1994 mit dem Nobelpreis im Bereich Ökonomie geehrt (Rossmüller 1994: 29).

Wie Ingaro und Israel (1990) in „The invisible hand: economic equilibrium in the history of science" darstellen, beförderte diese Idee der Analyse allgemeiner Gleichgewichtszustände durch die Aufnahme mathematischer Prinzipien zugleich die Quantifizierung ökonomischer Phänomene. Zugleich etablierte sich die Spieltheorie in den 50er und 60er Jahren zunehmend im Bereich der Evolutionsbiologie und der Politikwissenschaften und stellte in diesen Bereichen mathematische Lösung für komplexe Problemen bereit (Hykšová 2004: 53 f.). Damit erhöhte sich auch die Akzeptanz, Verbreitung und wissenschaftliche Anerkennung ihrer analytischen Modelle und Methoden für die Untersuchung empirischer Phänomene. Im Unterschied zu anderen wissenschaftlichen Theorien und Modellen erfolgte in der Spieltheorie kaum eine Abwandlung oder Anpassung der theoretischen Konzepte für die jeweilige Disziplin, in der sie angewendet wurde, sodass Konzepte wie das Nash-Gleichgewicht gleichermaßen in der Evolutionsbiologie wie auch in der Politikwissenschaft und Ökonomie für Untersuchungen genutzt wurden.

Noch in den 80er Jahren verwiesen die Ökonomen Paul A. Samuelson und William D. Nordhaus in ihrem internationalen Standardwerk „Economics“ (1985) darauf, dass die ökonomische Forschung sich vorrangig darauf verlegen musste, zu beobachten, da ihr die empirische Basis für die Verifikation ihrer Theorien fehle (ebd.: 8). Aufgrund dieser fehlenden empirischen Basis kann der operationale Charakter der ökonomischen Modelle in diesem Sinne nicht mit dem Vorgehen der Naturwissenschaften parallelisiert werden, da es ihnen an dem empirischen Nachweis der theoretisch aufgestellten Kausalitätsbeziehungen mangelt (Jonas 1964: 175). Wie Debreu (1991) in seinem Aufsatz „The Mathematization of Economic Theory" aufzeigt, stellte die Physik für die Ökonomie jedoch weiterhin ein unerreichbares Ideal dar. Der Grund war, dass man in der Physik neben der logischen Deduktion, die sich durch mathematische Modelle ergibt, zugleich auch auf empirische Beobachtungen durch die Ergebnisse von Experimenten zurückgreifen konnte. Im Unterschied dazu fehlten der Ökonomie die Möglichkeiten, empirische Daten durch Experimente zu erheben, sodass sie sich allein auf die deduktive Logik verlegen musste, um ihre Theorien zu belegen. Diese waren jedoch stets der Gefahr ausgesetzt, durch alternative Modelle an Glaubwürdigkeit zu verlieren (Debreu 1991: 2 f.). 


\subsubsection{Die Etablierung der experimentellen Methode}

Auch wenn die ersten ökonomischen Experimente bereits in den 1940er Jahren durchgeführt wurden, dauerte es mindestens weitere 40 Jahre bis sich ökonomische Experimente als wissenschaftliche Methode etablieren konnten. Während sich der empirische Teil dieser Arbeit der Analyse von Laborexperimenten widmet, wurden die ursprünglichen ökonomischen Experimente nicht in Laboren durchgeführt, sondern als ,Classroom-Experiment" in Seminar- beziehungsweise Vorlesungsräumen. Der Grundstein für zentrale methodologische Prinzipien, die auch heute noch das Vorgehen in Laborexperimenten prägen, wurde in diesen Experimenten gelegt.

Edward Chamberlin war wohl der erste Ökonom, der ein experimentelles Vorgehen einsetzte, um seinen Studierenden in Harvard aufzuzeigen, wie Marktpreise entstehen, wenn ein Markt durch Abweichungen von einem vollkommenen Wettbewerb charakterisiert ist (Smith 1992). Zu diesem Zweck teilte er seine Studierenden in KäuferInnen und VerkäuferInnen auf und teilte ihnen mit, dass es ihr Ziel sei, einen Handelspartner für den Kauf beziehungsweise Verkauf bestimmter Waren zu finden. Jeder Student in der Rolle eines Käufers erhielt eine Karte, auf welcher sein maximaler Ankaufspreis (pro Stückgut) ausgewiesen war. Analog dazu erhielten die VerkäuferInnen eine Karte, auf der der minimale Verkaufspreis vermerkt war. Nun sollten die Teilnehmenden im Seminarraum umherlaufen und nach möglichen Handelspartnern suchen und mit ihnen Preise aushandeln. Fand sich ein geeignetes Paar von KäuferInnen und VerkäuferInnen, so sollten sie einen ,Vertrag' schließen. Hierzu sollten sie ihre Karten an Chamberlin weitergeben und den Preis angeben, auf welchen sie sich geeinigt hatten. Chamberlin notierte diesen ausgehandelten Wert wiederum an der Wandtafel des Raumes. Da die festgehaltenen Werte aufzeigten, dass sich die Preise nicht dem vorhersagbaren Gleichgewichtspunkt näherten, betrachtete Chamberlin das Ergebnis des Experiments als empirische Grundlage für eine Kritik an der Wettbewerbstheorie (Chamberlin 1948: 95).

Einer der anwesenden Studierenden war 1952 Vernon Smith, der später zum Pionier für die Etablierung des Einsatzes von Experimenten in der wirtschaftswissenschaftlichen Forschung wurde. Wie Smith (1992) selbst ausführte, wurden die Experimente von Chamberlin als unwichtige Lehrvorführungen („classroom demonstration") oder Gesellschaftsspiele wahrgenommen. Sie wurden (auch von Smith selbst) als unwissenschaftlicher Weg betrachtet, auf dem Chamberlin versuchte, Widersprüche in der Theorie des perfekten Wettbewerbs zu demonstrieren. Das Potenzial von ökonomischen Experimenten wurde zu Smith Studentenzeiten noch nicht erkannt: „Perhaps the best evidence for this interpretation is 
that although many generations of Harvard graduate students were exposed to Chamberlin's classroom exercise, apparently I was the only one of them who ultimately carried further the idea of doing experiments in economics" (Smith 1992: 242). Die Ergebnisse und das Vorgehen Chamberlins wurden nicht als wissenschaftliches Experiment betrachtet und entsprachen einer methodologischen Außenseiterposition im Hinblick auf das gängige wirtschaftswissenschaftliche Vorgehen der 50er Jahre. Chamberlin selbst nahm seine Versuche hingegen sehr wohl als Experimente wahr, wie eine Publikation aus dem Jahr 1948 belegt. Hier verwies Chamberlin zwar sehr wohl auf die Gründe für die Begrenztheit gängiger ökonomischer Methoden, zeigte jedoch zugleich auf, welche Chance ein experimentelles Vorgehen für die ökonomische Forschung bot:

„It is a commonplace that, in its choice of method, economics is limited by the fact that resorts cannot be had to the laboratory techniques of the natural sciences. On the one hand, the data of real life are necessarily the product of many influences other than those which it is desired to isolate - a difficulty which the most refined statistical methods can overcome only in small part. On the other hand, the unwanted variables cannot be held constant or eliminated in an "economic" laboratory" because the real world of human beings, firms, markets, and governments cannot be reproduced artificially and controlled. The social scientist who would like to study in isolation and under known conditions the effects of particular forces is, for the most part, obliged to conduct his "experiment" by the application of general reasoning to abstract "models." He cannot observe the actual operation of a real model under controlled conditions. The purpose of this article is to make a very tiny breach in this position: to describe an actual experiment with a "market" under laboratory conditions and to set forth some of the conclusions indicated by it. The experiment has been carried out in a number of classes in economic theory, with the students offering themselves up as guinea pigs. It was actually designed to illuminate a particular problem which I had analysed earlier in abstract terms. " (Chamberlin 1948: 95)

Die Problematik der Anwendung von Experimenten auf ökonomische Phänomene, die Chamberlin hier aufzeigt, geht einher mit der Komplexität, die diesen Phänomenen in ihrer realweltlichen Existenz zugeschrieben wird. Wie weiter oben ausgeführt, stellt der wahrgenommene Komplexitätsgrad ökonomischer Phänomene den zentralen Grund dafür dar, dass von einer empirischen Überprüfung von Hypothesen und Modellen bisher abgesehen wurde. Auch Chamberlin erkennt an, dass keine realweltlichen Zusammenhänge im Experiment abgebildet werden können. Stattdessen verweist er jedoch auf das Experiment als einen Weg, abstrakte Modelle unter kontrollierten Bedingungen zu testen und so abstrakte Begriffe einer empirischen Prüfung zu unterziehen. Chamberlins Anwendung von Experimenten bezog sich somit auf den Einsatz als Werkzeug des Theorietestens. 
Als Smith Mitte der 50er Jahre selbst eine ökonomische Einführungsveranstaltung an der Purdue Universität gab, betrachtete er die damaligen Experimente von Chamberlin erneut und kam zu einem anderen Schluss hinsichtlich ihres Potenzials für die ökonomische Forschung: „Upon reconsideration it struck me that the idea of designing experiments to test a proposition derived from economic theory was right" (Smith 1992: 243). Die Art und Weise, wie Chamberlin die Marktsituation im Rahmen seines Experiments konzipiert hatte, war Smiths Ansicht nach auf zu vereinfachenden institutionellen Mustern aufgebaut, sodass er einige systematische Veränderungen vornahm. ${ }^{15}$ Im Januar 1956 setzte Smith sein Marktexperiment zum ersten Mal im Rahmen einer seiner Lehrveranstaltungen ein. Im Unterschied zu Chamberlin (1948) stellte Smith (1962) nun nach einer Reihe von Experimenten fest, dass sich in seinem Marktexperiment recht schnell ein Gleichgewichtszustand im Sinne eines Wettbewerbsgleichgewichts zwischen den Preisen von KäuferInnen und VerkäuferInnen einstellte. Die Abweichungen von diesem Gleichgewicht führte Smith auf zentrale Verfahrensgrundlagen der Experimente zurück, welche er als „Treatment-Effekte“"16 bezeichnete. In einem Fall, in welchem sich der Gleichgewichtzustand oberhalb des Wettbewerbsgleichgewichts eingependelt hatte, wiederholte Smith das Experiment und setzt zusätzlich einen finanziellen Anreiz. Er entlohnte die Teilnehmenden dabei für jede erfolgreiche Transaktion und jeden von ihnen erzielten Profit ${ }^{17}$ mit einem kleinen Geldbetrag. Der Einsatz eines finanziellen Anreizes führt laut Smith dazu, dass bereits in der dritten Spielrunde das Wettbewerbsgleichgewicht erreicht wurde und belegte für ihn eindeutig die entscheidende Relevanz finanzieller

\footnotetext{
${ }^{15}$ Die zentralen Veränderungen, die Smith vornahm, bestanden darin, dass alle VerkäuferInnen und KäuferInnen vollständige Informationen über alle Kauf- und Verkaufsangebote der anderen Teilnehmenden hatten. Zudem wurde die Marktumgebung so organisiert, dass die Angebots- und Nachfragepreise in bestimmten festgelegten Zeiträumen (Handelstag) abgegeben werden mussten, wobei das Experiment mehrere solcher Zeitintervalle beziehungsweise Runden umfasste.

${ }^{16}$ Die Differenz zwischen unterschiedlichen Versuchsanordnungen (Treatments) ist ein grundlegendes Merkmal des experimentellen Vorgehens, welches sich definitionsgemäß einem Vergleich zwischen Kontroll- und Untersuchungsgruppe widmet (siehe Abschnitt 2.1). Treatment-Effekte bezeichnen im hier verwendeten Sinne jedoch den ungewollten beziehungsweise unreflektierten Einfluss auf Ergebnisse von Experimenten, die sich durch die Versuchsanordnung des Experiments ergeben. Für diese unintendierten Verzerrungen wird in späteren methodologischen Diskussionen der Begriff der Framing-Effekte (siehe Abschnitt 5.2.2.1) verwendet.

${ }^{17}$ Der Profit eines Teilnehmenden bezeichnet hier die Differenz zwischen dem minimalen Verkaufspreis bzw. dem maximalen Kaufpreis und dem in der Transaktion tatsächlich gewählten Wert.
} 
Anreize bei der Durchführung von Experimenten. „This established clearly for me that monetary rewards mattered, and I never again conducted an experiment without using rewards that exceeded the opportunity cost of the typical subject" (Smith 1992: 244). Zentrale Grundlagen und methodologischen Reflexionen über Laborexperimente bauten auf diesen ersten Classroom-Experimenten auf. Im obigen Zitat zeigt sich dabei ein deutlicher Hinweis auf die Bedeutung, die Smith der Monotonie eines wirkungsvollen Anreizsystems im allgemeinen und spezifischen monetären Anreizen im Rahmen von Experimenten beimisst. Eben diese Monotonie des Nutzens bildet ein Grundprinzip von Smiths ,,induzierter Werttheorie" (Induced Value Method) (siehe Abschnitt 5.2.2.2), welche bis heute ein elementares methodologisches Fundament und ein Paradigma der experimentellen ökonomischen Laborforschung darstellt.

Ab den 1950er Jahren schloss sich in Princeton eine Gruppe von Mathematikern (u. a. John Nash) zusammen, die eine empirische Tradition des „Gaming“ begründete, welche spieltheoretische Annahmen durch empirische Versuche überprüfte oder illustrierte. Eine zweite einflussreiche Kooperation zeigte sich an der RAND Corporation in Santa Monica ${ }^{18}$ in einer Forschergruppe, die hauptsächlich aus Psychologen und Mathematikern bestand. Einen weiteren zentralen Meilenstein in der Etablierung der experimentellen Ökonomie stellte 1952 die interdisziplinäre Konferenz „The Design of Experiments in Decision Processes“ in Santa Monica dar, die interessierte Mathematiker, Ökonomen und Psychologen versammelte (Sunder \& Friedman 1994: 124; Smith 1992: 260 ff.). Auch wenn das allgemeine Thema dieser Konferenz eher in der Nutzung mathematischer Methoden für sozialwissenschaftliche Fragestellungen lag, widmeten sich 5 von 19 Vortragspapieren und ein Großteil der Diskussion der Darstellung und Interpretation der Ergebnisse von Experimenten. Einer der Teilnehmer war Herbert Simon. Aufbauend auf seinen dort vorgestellten Ergebnissen, stellte Simon eine Differenz zwischen dem fest, was für den Experimentierenden als rational erscheint und dem, was für die Versuchspersonen rational ist ${ }^{19}$ und legte damit einen wichtigen Grundstein für sein Konzept der begrenzten Rationalität (siehe Abschnitt 5.2.1).

\footnotetext{
${ }^{18}$ Bei der RAND Cooperation Santa Monica handelt es sich um eine 1948 gegründete Forschungs- und Entwicklungseinrichtung, die sich mit politiknahen Themen befasste und anfänglich vor allem auf militärische Fragestellungen ausgerichtet war.

${ }^{19}$ Wie aus den Ausführungen in Abschnitt 2.1 deutlich geworden sein sollte, besteht zwischen Forschenden und Versuchsteilnehmenden eine Informationsasymmetrie. Während die Forschenden über vollständige Informationen über das experimentelle Setting und Verfahren verfügen, ist das Wissen der ProbandInnen über die Entscheidungssituation üblicherweise unvollständig und begrenzt (Smith 1992: 260f; Sunder \& Friedman 1994: 125)
} 
Die Auseinandersetzung zwischen Psychologen, die disziplinär bereits auf eine lange experimentelle Tradition zurückblicken konnten, und ÖkonomInnen, StatistikerInnen und MathematikerInnen, die dieses Feld nun neu für sich entdeckten, führte zu fruchtbaren Reibungen hinsichtlich der methodologischen Grundlagen bei der Anwendung von ökonomischen Experimenten. Auch wenn Smith als die zentrale Schlüsselfigur für den Aufbau der Methodologie der experimentellen Wirtschaftswissenschaften gilt, verweist dieser selbst explizit darauf, dass zentrale Prinzipen des experimentellen Forschungsdesigns in entscheidendem Maße auf Arbeiten des Psychologen Sidney Siegel zurückgehen (Smith 1992: 247). ${ }^{20}$ So zeigte Siegel anhand eigener Studien auf, dass die Rahmenbedingungen der Experimente maßgeblich das Ergebnis der Experimente beeinflussen und aus diesem Grund einer gezielten Kontrolle und Reflexion unterzogen werden müssen. Das schriftliche Festhalten der Instruktionen, die Nutzung von (finanziellen) Anreizen zur Motivation, die Relevanz von Langeweile und Monotonie und viele weitere methodologisch relevante Aspekte wurden dabei von Sigel thematisiert und empirisch untersucht (ebd.).

Auch wenn die experimentelle Methode eine gewisse Resonanz fand, erschien sie noch lange Zeit als eher unüblicher Sonderweg, wie Smith (1992) in einer Selbstreflexion seines wissenschaftlichen Werdegangs angibt. Dies änderte sich erst Ende der 1970er Jahre. Zunehmend konnte belegt werden, dass durch Experimente nicht nur für die Ökonomie, sondern auch für die öffentliche Wirtschaft und auch für andere Bereiche der Politikwissenschaft entscheidende und neue Erkenntnisse ermöglicht wurden (beispielsweise Fiorina \& Plott 1978; Plott \& Levine 1978; Plott \& Smith 1978). In zwei fundamentalen Texten fasste Smith die methodologischen Grundzüge der experimentellen Wirtschaftsforschung zusammen (Smith 1976; Smith 1982). In den 1980er Jahren nahm die Akzeptanz für die experimentelle Wirtschaftsforschung sprunghaft zu; Forschung, Projekte und Publikationen in Bereich der experimentellen Ökonomie steigerten sich enorm. Damit einher ging auch die Gründung erster größerer Forschungszentren in den 1990er Jahren (z. B. in Arizona, Caltec und Bonn) und spezifischer Fachzeitschriften (z. B. Experimental Economics 1998).

\footnotetext{
${ }^{20}$ Siegel und Smith lernten sich erst im Herbst 1961 kennen und bereits Ende des Jahres verstarb Siegel an einem Herzinfarkt. Wie Smith selbst darstellt, hatte diese kurze Zeit jedoch einen entscheidenden Einfluss auf methodologische Aspekte der sich entwickelnden experimentellen ökonomischen Forschung: „Sid Siegel's concern for the content of instructions, his insistence that instructions should be part of one's report, his practice of always motivating subjects with meaningful cash rewards, and much more are all evident in this work [...] and in the work of experimental economists today." (Smith 1992: 247)
} 
Die Entwicklung der experimentellen Wirtschaftsforschung in Deutschland begann ebenfalls in den 1950er Jahren und geht maßgeblich auf die Initiative von Richard Selten zurück. Wie bereits im Abschnitt über die Entwicklung der Spieltheorie ausgeführt, stieß Selten zentrale Weiterentwicklungen im Bereich des Nash-Gleichgewichtes an. Bereits in seiner ersten Publikation 1960 nutze er ein experimentelles Vorgehen für die empirische Validierung seines theoretischen Ansatzes (Sauermann \& Selten 1960). In der Buchreihe „Beiträge zur experimentellen Wirtschaftsforschung" (Sauermann 1967, 1970, 1972) wurden zentrale Papiere gesammelt und einer breiteren Öffentlichkeit zugänglich gemacht. Mit der Gründung der „Gesellschaft für experimentelle Wirtschaftsforschung“21 wurde 1977 eine Vereinigung geschaffen mit dem expliziten Ziel, die experimentelle Methodik in der Ökonomie zu etablieren. Im Unterschied zur experimentellen Wirtschaftsforschung in den USA, konzentrierten sich die Arbeiten der deutschen Forscher anfänglich vorrangig auf die Betrachtung von Spiel- und Entscheidungssituationen und weniger auf Marktsituationen. Eine zentrale Orientierung stellte Simons Theorie der begrenzten Rationalität (siehe Abschnitt 5.2.1.2) dar, mittels derer man versuchte, menschliche Verhaltensprozesse in ökonomisch relevanten Situationen zu erklären.

Aufgrund mangelnder empirischer Evidenz waren ökonomische Experimente bis in die 1980er Jahre nicht dazu geeignet, nachzuweisen, ob grundlegende mikroökonomische Annahmen wie die Nutzenmaximierung der Akteure oder Theorien von Marktgleichgewichten die empirische Realität richtig erfassten. Ihr entscheidender Wert lag vielmehr in einer begründeten Auswahl zwischen verschiedenen konkurrierenden Theorien. Wie oben beschrieben, wurde beispielsweise im Rahmen der Spieltheorie das Konzept von Spielgleichgewichten entwickelt und durch Nash und andere Wissenschaftler weiter ausgebaut. Es entwickelten sich unterschiedliche theoretische Gleichgewichtsmodelle. Je nach konzeptionellen Annahmen über die Präferenzen und den Informationstand der beteiligten Akteure konnte es auch vorkommen, dass im Rahmen eines Modells unterschiedliche Gleichgewichtspunkte möglich waren. Die unterschiedlichen konkurrierenden Theorien und Lösungsvorschläge verfügten dabei jeweils über in sich logisch konsistente und plausible Annahmen für die Prognose oder Herstellung dieser Gleichgewichtspunkte. Es mangelte den spieltheoretischen Modellen jedoch an empirischen Evidenz. Mittels von Experimenten bot sich nun die Chance, begründete Argumente für oder gegen spezifische theoretische Positionen und Annahmen bereitzustellen. Die leitende Frage war dabei stets, welches

\footnotetext{
${ }^{21}$ Weitere Informationen finden sich auf der Homepage der „Gesellschaft für experimentelle Wirtschaftsforschung“: https://www.gfew.de/ (Zugriff am 26.08.2018)
} 
theoretische Konzept die Ergebnisse eines einfachen Experiments am besten vorhersagen konnte (Plott 1991: 906). Erst im Zuge eines theoretischen und konzeptionellen Pluralismus lag also die Chance, die Methode des Experiments zu etablieren.

Mittlerweile ist die experimentelle Wirtschaftsforschung ein fester Bestandteil der Wirtschaftswissenschaften. Im Rahmen der Mikroökonomie hat dieses methodische Vorgehen, ebenso wie die Spieltheorie, Eingang in den universitären Lehrplan und die Ausbildung von Studenten gefunden. Wie die Ausführungen zeigten, konnte grundlegende ökonomische Prinzipien bereits durch die Classroom-Experimente empirisch begründet und veranschaulicht werden. Während diese Experimente anfangs mit einfachen Mitteln durchgeführt wurden, setzte sich zunehmend die Datenerhebung in speziell dafür konzipierten Laboren durch. Solche Experimentallabore finden sich heutzutage an vielen Universitäten und außeruniversitären Forschungseinrichtungen. Es existieren zudem zahlreiche Lehr- und Handbücher, die in die Praxis der experimentellen Forschung einführen und den Stellenwert der ökonomischen Experimente bezeugen (Hey 1991; Sunder \& Friedman 1994; Davis \& Holt 1993; Kagel \& Roth 1995, 2016).

\subsubsection{Gründe für den Einsatz von Experimenten in der ökonomischen Forschung}

Im letzten Abschnitt dieses Einführungskapitels soll nun überblicksartig dargestellt werden, aus welchen Gründen Laborexperimente in den Wirtschaftswissenschaften genutzt werden. Hinsichtlich der Unterscheidung der Nutzungsgründe von Experimenten in der ökonomischen Forschung wird hier die von Alvin Roth (1986) vorgeschlagene Differenzierung in drei zentrale Funktionen übernommen: „Speaking to theorists“, „Searching for facts“, „,Whispering in the ear of princes". 22

Wie oben dargestellt, wurden Experimente im Bereich der Wirtschaftswissenschaften zu Beginn vor allem zum Testen von (konkurrierenden) Theorien und ihrer konzeptuellen Annahmen eingesetzt. ${ }^{23}$ Roth bezeichnet diese Funktion als

\footnotetext{
${ }^{22}$ Eine etwas andere Sortierung nimmt Smith (1994) vor, da er die erste Ebene des Theorievergleichs noch weiter ausdifferenziert. Insgesamt zeigt sich jedoch auch hier das gleiche Spektrum am Einsatzbegründungen für Laborexperimente in der Wirtschaftswissenschaft.

${ }^{23}$ In einem Interview mit Vernon Smith (Smith 1992: 257) gibt James Friedman, eine weitere historische Ikone im Bereich der experimentellen Wirtschaftsforschung, eine typische Begründung für diese Art der Nutzung von Experimenten in der Forschung: „For me the great motivation and prime interest lies in testing the predictions of economic theory. When
} 
„Speaking to theorists“ (Roth 1986: 246). Der Grund für den Einsatz von Experimenten besteht also darin, dass deskriptive Theorien der ökonomischen Forschung und die ihnen zugrundeliegenden Annahmen nur schwerlich einer empirischen Prüfung unterzogen werden können. Die Differenz zwischen dem natürlichen Auftauchen eines empirischen Phänomens und seiner theoretischen Modellierung stellt eine zentrale methodische Herausforderung für die ökonomische Forschung dar. Aus diesem Grund können unterschiedliche Theorien und Erklärungsversuche konkurrierend nebeneinander stehen, ohne dass entschieden werden kann, welche Theorie die empirischen Phänomene valider erklärt. Mit Hilfe von Experimenten kann nun unter kontrollierten Bedingungen die Stichhaltigkeit von bestimmten theoretischen Annahmen überprüft werden, um so die Grundlagen der theoretischen Konzepte und Annahmen abzusichern. Das Design der Experimente soll es dabei erlauben, ganz gezielt bestimmte Annahmen von Theorien zu testen. Dies wird insbesondere in den Fällen als großer Vorteil erachtet, in denen die getesteten Annahmen sehr sensibel auf das Entscheidungsumfeld reagieren oder schwierig zu beobachten sind. Das experimentelle Setting wird dabei so konzipiert, dass durch den Einsatz bestimmter Darstellungsmittel gezielt die Wirkung von einzelnen Einflussfaktoren ausgeschaltet werden soll; zugleich werden andere Aspekte isoliert und damit manipulier- und messbar gemacht (Roth 1986: 246 f.). Diese Art von Experimenten dient somit vorrangig der Grundlagenforschung und dem Vergleich von Theorien. An dieser Stelle zeigt sich wiederum, dass der Auswahl und dem Einsatz von geeigneten Darstellungsmitteln konzeptionelle Annahmen und methodologische Traditionen vorausgehen, die beeinflussen, welche Darstellungsmittel zur Abbildung oder Ausschaltung bestimmter Einflussfaktoren eingesetzt werden.

you test a theoretical prediction on naturally occurring data you do not find out whether the prediction will hold under the assumptions of the model from which the prediction comes. The reasons are that naturally occurring data inevitably arise in circumstances that don't satisfy the conditions of our models and the correct conditions are imperfectly known. What you find may be valuable in making policy, but the foundations are shaky when you lack good knowledge of the underlying model that generates the naturally occurring data. You don't have a clear picture of why the given prediction works (or fails to work, as the case may be). To build theory from solid foundations and to build a strong structure, I believe that testing predictions in conditions that satisfy the assumptions under which the predictions are made is the first step in gauging the validity of such predictions. This sort of testing does not imply that the same predictions will obtain on natural data; however, it is an essential step in seeing whether a theory is valid on its own terms." Dass Entscheidungssituationen im Labor als modellierbar angenommen werden, betrachtet Friedman somit als den zentralen Vorteil für das Testen von Theorien und Modellannahmen. Auf diese Weise könne die interne Güte oder Validität der Annahmen in einem ersten Schritt selbst überprüft werden, bevor sie im Folgenden zur Analyse realweltlicher Zusammenhänge eingesetzt werden. 
Es ist jedoch nicht ungewöhnlich, dass im Zuge von Experimenten auch relevante Aspekte und Einflussfaktoren entdeckt werden und erst auf diesem Weg Eingang in die ökonomische Theorie finden. Roth spricht in diesem Zusammenhang von ,Searching for facts“ und verweist auf den Beitrag, den die durchgeführten Laborexperimente zur Theoriebildung und deren Weiterentwicklung beitragen. Prominent sind in diesem Bereich Untersuchungen zum Thema Fairness, Vertrauen oder Altruismus in Entscheidungssituationen (Überblicksartikel von Fehr \& Gächter 2000; Fehr \& Schmidt 2001; Levit \& List 2007). Diese Aspekte stehen häufig im Widerspruch zu den klassischen ökonomischen Annahmen hinsichtlich des zentralen Stellenwerts der Maximierung des Eigennutzens, an welchen sich Handelnde im Sinne des Modells des Homo oeconomicus orientieren sollen. Diese Effekte konnten jedoch wiederholt in Experimenten nachgewiesen werden und motivierten zur Entwicklung neuer Forschungsprojekte und theoretischer Ansätze. Mit Hilfe von Experimenten können in diesem Sinne auch Phänomene sichtbar und analytisch erfassbar gemacht werden, die in einer natürlichen Umgebung in ,Reinform“ nicht beobachtbar sind (Hacking 1999: 135). Es handelt sich dabei vielfach um Themen und Aspekte, die klassischerweise der psychologischen Forschung zugeordnet werden. Der Bereich der Verhaltensökonomie macht nun ebendiese Analyse psychologischer Aspekte auf das ökonomische Entscheidungsverhalten zu seinem zentralen Untersuchungsgegenstand.

Die dritte Funktion, auf die Roth verweist, „Whispering in the ear of princes“, nimmt Bezug auf die Potenziale, welche die experimentelle Forschung im Bereich der Politikberatung oder zu Prognosezwecken entfaltet. Der „Iowa Electronic Market" ist ein bekanntes Beispiel. Es handelt sich hierbei um eine Internetplattform der Universität Iowa, auf der Aktienmarktexperimente durchgeführt werden, die sich primär mit der Prognose von Wahlergebnissen befassen. ${ }^{24} \mathrm{Im}$ Bereich der Politikberatung werden Experimente zudem eingesetzt, um mögliche Marktmechanismen zu testen und zu überprüfen, welche am besten den Anforderungen des jeweiligen Marktes entsprechen. ${ }^{25}$ Um diese Funktion zu erfüllen, müssen die Experimente jedoch fähig sein, bei der Komplexitätsreduktion, die

\footnotetext{
${ }^{24}$ Der Zugang zur Internetplattform ist über folgenden Link möglich: https://tippie.biz.uiowa. edu/iem/. (Zugriff am 05.11.2018)

${ }^{25}$ Relevante Dimensionen sind dabei beispielsweise die effiziente Verteilung von Wettbewerbspreisen und zur Verfügung gestellten Ressourcen, die Einfachheit des Verfahrens für die Teilnehmenden oder die für die Auktion benötige Zeit. Auch im Bereich der MatchingMärkte, bei denen die Verteilung nicht über Preise bestimmt wird, erweist sich die Anwendung von Experimenten als hilfreich für die Analyse und Beratung politischer Prozesse und die Überprüfung der Eignung des Marktmechanismus. Beispielhaft kann hier auf die Studie von John H. Kagel und Alvin E. Roth (2000) zum Arbeitsmarkt für junge Ärzte in England oder
} 
mit dem Experiment einhergeht, die relevanten Dimensionen dennoch abzubilden. Dieser Bereich der Nutzung experimenteller Ergebnisse und insbesondere der verhaltensökonomischen Forschung wird aktuell prominent unter dem Schlagwort „Nudging“ (dt. Schubsen oder Stupsen) diskutiert. In starker Anlehnung an die Schriften der Psychologen Daniel Kahneman und Amos Tversky (Kahneman 2011; Kahneman et al. 1982; Kahneman \& Tversky 2000; Tversky \& Kahneman 1974) wird darauf verwiesen, dass die Ergebnisse der experimentellen Verhaltensforschung belegen, dass Handelnde bei ihren Entscheidungen häufig irrational agieren und auf Heuristiken oder Daumenregeln zur Bewertung ihrer Entscheidungssituation zurückgreifen. Zugleich führt eine Veränderung der Anreizstruktur oder der Problemdarstellung häufig zu einer Veränderung der wahrgenommenen Entscheidungsalternativen ${ }^{26}$ und motiviert somit zu anderen Entscheidungen. Durch einen kleinen Schubs soll es nun gelingen, die Handelnden zu einem ,besseren " und ,rationaleren' Verhalten zu motivieren. Eine deutliche Verbreitung und Popularisierung dieser Ideen erfolgte in den letzten Jahren durch Kahnemans Bestseller „Thinking, fast and slow“ (2011) und Richard Thalers und Cass Sunsteins Buch „Nudge“ (2008). Thaler und Sunstein zeigen anhand von verschiedenen Beispielen auf, wie Nudging im Sinne eines ,libertären Paternalismus“ (Thaler \& Sunstein 2008) zur Lösungen oder Verbesserungen bestimmter gesellschaftlicher Problembereiche wie der Organspende beitragen könnte. ${ }^{27}$ Neben Feldexperimenten bilden auch in diesem Fall Laborexperimente einen zentralen Bestandteil der empirischen Daten, auf denen die verhaltensökonomischen Erkenntnisse gründen.

Das Ziel des zweiten Kapitels bestand in einer thematischen Einführung in den untersuchten Gegenstandsbereich. Dies erfolgte einerseits durch die Darstellung grundlegender methodologische Aspekte der allgemeinen Verwendung von Experimenten in den Sozialwissenschaften, andererseits durch die Ausführungen

die Studie von Braun et al. (2010) zum zentralen Zuweisungsmechanismus für Studienplätze im Bereich der Medizin in Deutschland verwiesen werden (Kübler 2015).

${ }^{26}$ Unter Rückgriff auf die Publikationen von Kahneman und Tversky (2000) wird dieses Thema primär unter dem Begriff des Framing beziehungsweise der Framing-Effekte diskutiert. Eine ausführliche Darstellung findet sich im Analyse Abschnitt 5.2.2.1 zur Außenstruktur des ökonomischen Laborexperiments.

${ }^{27}$ Ein bekanntes Beispiel ist das Organspendeverhalten, das die Autoren ausführlich diskutieren. Während man sich in Deutschland aktiv für einen Organspendeausweis anmeldet (Zustimmungsreglung), werden in anderen Ländern wie Österreich oder Portugal erst einmal alle als Organspender behandelt, außer sie erheben dagegen Widerspruch und melden sich ab (Widerspruchsregelung). Der Vergleich von europäischen Ländern, in denen die Widerspruchsregelung genutzt wird, zeigt dabei im Vergleich eine deutlich höhere Spenderquote. 
zur Etablierung der experimentellen Methode in der wirtschaftswissenschaftlichen Forschung und die feldinterne Begründung für den Einsatz von Laborexperimenten. In einem nächsten Schritt wird nun im Kapitel 3 die theoretische und konzeptionelle Perspektive spezifiziert, aus welcher die Erforschung des Phänomens des ökonomischen Laborexperiments erfolgte.

Open Access Dieses Kapitel wird unter der Creative Commons Namensnennung 4.0 International Lizenz (http://creativecommons.org/licenses/by/4.0/deed.de) veröffentlicht, welche die Nutzung, Vervielfältigung, Bearbeitung, Verbreitung und Wiedergabe in jeglichem Medium und Format erlaubt, sofern Sie den/die ursprünglichen Autor(en) und die Quelle ordnungsgemäß nennen, einen Link zur Creative Commons Lizenz beifügen und angeben, ob Änderungen vorgenommen wurden.

Die in diesem Kapitel enthaltenen Bilder und sonstiges Drittmaterial unterliegen ebenfalls der genannten Creative Commons Lizenz, sofern sich aus der Abbildungslegende nichts anderes ergibt. Sofern das betreffende Material nicht unter der genannten Creative Commons Lizenz steht und die betreffende Handlung nicht nach gesetzlichen Vorschriften erlaubt ist, ist für die oben aufgeführten Weiterverwendungen des Materials die Einwilligung des jeweiligen Rechteinhabers einzuholen.

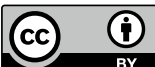

